\title{
DiE VIELEN FACETTEN DER VULNERABILITÄT IM KONTEXT VON FLUCHT UND ASYL
}

\author{
Gabriele RaSUly-PALECzeK, Institut für Kultur- und Sozialanthropo- \\ logie, Universität Wien
}

\section{INHALT}

1 Einleitung

2 Das Vulnerabilitätsparadigma im operativen Flüchtlings- und

Migrant/inn/enschutz.

3 Das Vulnerabilitätsparadigma in der Flucht- und Migrationsforschung ................... 43





\section{Einleitung}

„All refugees are vulnerable“

Ich möchte dieses Statement von Michel AgIER (2011, p. 158) aus seinem Buch „Managing the Undesirables. Refugee Camps and Humanitarian Government“ zum Anlass nehmen, um in diesem Beitrag einen kurzen Überblick über den gegenwärtigen Diskurs rund um das Vulnerabilitätsparadigma in Politik und Fluchtforschung zu geben. Nach einigen allgemeinen Erläuterungen zum Begriff Vulnerabilität wird zunächst auf die Verwendung des Begriffs im Kontext des operativen Schutzes von Flüchtlingen und Migrant/inn/en (z.B. IOM, UNHCR) eingegangen und danach werden verschiedene Forschungsansätze zum Thema Vulnerabilität von Flüchtlingen und Migrant/inn/en näher vorgestellt. 
Der Begriff Vulnerabilität leitet sich vom lateinischen vulnus „Wunde, Verletzung, Schaden „vulnerare “ „verwunden, verletzen“ ${ }^{\text {"1 }}$ ab und beschreibt, wie LoRENz feststellt „... die Anfälligkeit von Bezugseinheiten oder Strukturen, welcher Art auch immer, Schäden durch äußere Einwirkung zu nehmen“ (Lorenz 2018, p. 62). Vulnerable zu sein ,... means to be exposed to the possibility of being harmed, either physically or emotionally“ (Oxford Dictionary² zitiert nach ATAK et al. 2018, p. 2).

Im Allgemeinen wird zwischen verschiedenen Formen der Vulnerabilität, z.B. physikalischer, technischer, infrastruktureller und sozialer Vulnerabilität differenziert (vgl. LoRENZ 2018, pp. 62 f.), wobei mit letzterer ,... die spezifische Anfälligkeit von Menschen, v. a. bezogen auf Konflikte, Katastrophen und Zwangsmigration, aber auch andere (kollektive) Stresssituationen gemeint (HEESEN et al. 2014) [ist]“ (LoRENZ 2018, p. 63).

Als „vulnerable Personen“ gelten daher Individuen „....with limited or reduced capacity, power, or control to protect their interests relative to other agents. From a biological or physiological perspective, vulnerability refers to a person's inherent characteristics and to a lack of means to cope without damaging loss“ (ATAK et al. 2018, p. 2). Oder wie CHAMBERs (2006, p. 33) konstatiert: „Vulnerability here refers to exposure to contingencies and stress, and difficulty in coping with them. Vulnerability has thus two sides: an external side of risks, shocks, and stress to which an individual or household is subject; and an internal side, which is defencelessness, meaning a lack of means to cope without damaging loss. Loss can take many forms becoming or being physically weaker, economically impoverished, socially dependent, humiliated or psychologically harmed.“

Die Vulnerabilität selbst kann auf unterschiedliche Weise verursacht sein. MackenZIE et al. (2014b) haben eine Taxonomie unterschiedlicher Quellen der Vulnerabilität vorgeschlagen. Sie unterschieden u.a. zwischen „inherent vulnerability“ and „situational vulnerability“.

„Inherent vulnerability' refers to sources of vulnerability that are intrinsic to the human condition. These vulnerabilities arise from our corporeality, our neediness, our dependence on others, and our affective and social natures. We are all inherently vulnerable to hunger, thirst, sleep deprivation, physical harm, emotional hostility, social isolation, and so forth“ (MACKENZIE et al. 2014a, p. 7). Einige dieser Vulnerabilitäten sind immer vorhanden, während andere von einer Reihe von Faktoren, wie z.B. Alter, Geschlecht und Gesundheitszustand, abhängen. Beispielsweise bewirkt ein schlechter Gesundheitszustand eine spezifische Vulnerablität, die in einem engen Zusammenhang mit der entsprechenden Erkrankung steht (vgl. ebenda). „Inherent vulnerability also varies depending on a person's resilience and capacity to cope“ (ebenda).

1 Übersetzungen für vulnus aus: < https://de.pons.com/\%C3\%BCbersetzung/latein-deutsch/ vulnus>; Übersetzung von vulnerare aus: <https://de.langenscheidt.com/latein-deutsch/vulnerare $>$.

$2 \quad$ Vgl. $<$ https://en.oxforddictionaries.com/definition/vulnerable; Zugriff 10-02-2018>. 
Im Gegensatz dazu ist die „situational vulnerability“ kontextspezifisch. Sie „... may be caused or exacerbated by the personal, social, political, economic or environmental situations of individuals or social groups. Situational vulnerability may be short term, intermittent, or enduring“ (MACKENZIE et al. 2014b, p. 7). Sie inkludiert, wie ATAK et al. (2018, p. 3) mit Bezugnahme auf MAcKenzIE et al. 2014b betonen, „... abusive relationships and oppression or injustice. This category highlights the ways in which inequalities of power, dependency, capacity, or need render some individuals vulnerable to harm or exploitation by others.“ Beide Formen der Vulnerabilität können „dispositional/potentiell“ oder „occurrent/aktuell“ sein (ebenda).

„For instance, women, children or migrants with disabilities are dispositionally vulnerable to exploitation. But whether or not these groups of migrants are actually vulnerable to exploitation will depend on a range of factors, such as their socioeconomic status or geographical location.“ (ebenda). Diese Unterscheidung zwischen potentieller und aktueller Vulnerabilität ist, wie ATAK et al. konstatieren, wichtig, ,... since it helps identify the sources of vulnerability in a particular case“ (ebenda). Vulnerabilität wird - wie kurz ausgeführt wurde - häufig zu spezifischen Risikofaktoren in Beziehung gesetzt, die in bestimmten Situationen schwerwiegende Konsequenzen haben können. „Vulnerability undermines autonomy, curtails individual capabilities or creates a sense of powerlessness“ (ebenda) oder wie HofFmaster (2006 zitiert nach ATAK et al. 2018, p. 3) feststellt: „... vulnerability means loss to all three urges human beings have: loss of opportunities to live better, loss of abilities to live well, and, at its extreme, loss of living.“

Allerdings sind nicht alle Personen in gleicher Weise von einem Konflikt, einer Katastrophe etc. betroffen, sondern es bestehen häufig sehr ,... unterschiedliche Anfälligkeiten und Betroffenheiten von Menschen“ (LoREnz 2018, p. 63) und nicht alle „vulnerablen Personen“ sind einfach nur „machtlose Opfer“ von Krisen, Stressfaktoren etc., sondern besitzen oft auch Resilienz und Agency (vgl. Details dazu Ausführungen unten). ${ }^{3}$

Das Vulnerabilitätsparadigma selbst wurde ursprünglich in der Katastrophenforschung entwickelt und fand später - um zahlreiche Ansätze angereichert - Eingang in andere Disziplinen, wie z.B. die Friedens-, Konflikt- und Sicherheitsforschung (vgl. LORENZ 2018, pp. 64 ff.). Seit einigen Jahren erfährt das Konzept der Vulnerabilität

3 In neuerer Zeit wird sowohl von Seiten internationaler Organisationen (z.B. IOM, UNHCR) als auch von zahlreichen Forscher/inne/n diese Vorstellung von „vulnerablen Personen“ als „powerless victims“ abgelehnt, sondern betont, dass diese über Resilienz und Agency verfügen (vgl. UNHCR et al. 2016, p. 2; IOM 2017; Савот 2014, pp. 110-142; CraWley et al. 2011; Krause \& Schmidt 2019; Brigden \& MaINWARING 2016; Baird 2014). 
zunehmend Anwendung in der Flucht- und Migrationsforschung, z.B. zur Charakterisierung der besonderen Situation von Geflüchteten ${ }^{4}$ und illegalen Migrant/inn/en. ${ }^{5}$

Das Vulnerabilitätsparadigma hat zudem in den Debatten und programmatischen Erklärungen diverser internationaler Organisationen (z.B. UNHCR, IOM) rund um den Schutz von Flüchtlingen und Migrant/inn/en eine beträchtliche Popularität erlangt (vgl. Lorenz 2018, p. 66; AtaK et al. 2018, p. 1; HruschKa \& Leboeuf 2019, p. 2). Beispielsweise kommt der Terminus ,vulnerability of migrants“ fünfzehnmal in der sogenannten „New York Declaration for Refugees and Migrants“ der UN Generalversammlung vom 19. September 2016 vor (vgl. AtaK et al. 2018, p. 1).

Insgesamt hat sich das Vulnerabilitätsparadigma sowohl in der Wissenschaft wie auch in der operativen Flüchtlings- und Migrant/inn/en/arbeit „... über die Zeit stark pluralisiert, so dass heute nicht mehr von einem einzelnen Ansatz gesprochen werden kann, sondern vielmehr eine Vielzahl von Verständnissen, Ansätzen und Methoden [existieren]“ (LoRENZ 2018, p. 63). Neben unterschiedlichen theoretischen Ansätzen und kritischen Diskursen zum Vulnerabilitätsbegriff (vgl. BANKOFF 2001, 2007; ATAK et al. 2018; Brown et al. 2017; Chambers 1989, 2006; Stewart 2005; Mackenzie et al. 2014a; Yaghmaian 2016) gibt es mittlerweile „... unzählige quantitative Indikatoren(systeme), Indizes und Kartierungen von Vulnerabilität ...“ (Lorenz 2018, p. 63), weswegen z.B. Susan L. CutTER (2003, p. 1) sogar von einer eigenständigen „vulnerability science“ spricht.

Obwohl der Begriff der Vulnerabilität in der Zwischenzeit sowohl in der Wissenschaft als auch in der Politik zu einem häufig verwendeten Schlagwort geworden ist, herrscht bislang kein Konsens darüber, was unter Vulnerabilität tatsächlich zu verstehen ist bzw. welche Personengruppen als vulnerabel gelten (vgl. HruschKa \& LeBoeuF 2019, p. 2; AтAK et al. 2018, pp. 1 f.; Lorenz 2018 und Brown et al. 2017, p. 497). Insgesamt bleibt seine Verwendung vage. Häufig wird der Begriff zudem als Synonym für Armut verwendet, wobei wie Chambers (2006, p. 33) konstatiert „Vulnerability, though, is not the same as poverty. It means not lack or want, but defencelessness, insecurity, and exposure to risk, shocks and stress.“

Des Weiteren findet der Begriff Vulnerability häufig Anwendung „... as an entry point for discussing inequality or adversities of some kind, with the concept draw on to anchor consideration of diverse interests and concerns. Prominent amongst these are insecurity, relative economic or social disadvantage, limited coping capacity and unmet need“ (Brown et al. 2017, p. 497).

Im Folgenden sollen einige Konzeptionen der Vulnerabilität sowohl aus dem operativen Flüchtlings- und Migrant/inn/enschutz als auch aus der Flucht- und Migrationsforschung vorgestellt werden.

$4 \quad$ Siehe z.B. Betts \& Loescher 2011 und Lorenz 2018, p. 66, für die bereits die bloße Existenz Geflüchteter Ausdruck von Vulnerabilität ist. Für das IOM besteht bezüglich der Vulnerabilität von Migrant/inn/en ein enger Konnex zwischen „Human Trafficking“ und Forced Labour (vgl. auch CLARK 2013 und DAVID et al. 2019).

5 Vgl. Han 2018; Lewis et al. 2015; Lorenz 2018; Pervou 2017; Stewart 2005; Askola 2010; GoldRING \& LANDOLT 2011. 


\section{Das Vulnerabilitätsparadigma im operativen Flücht- lings- und Migrant/inn/enschutz}

Angesichts steigender Flüchtlings- und Migrant/inn/enzahlen, die auf eine Vielzahl unterschiedlicher Gründe (z.B. langdauernde militärische Auseinandersetzungen, Klimawandel, Umweltkatastrophen etc.) zurückzuführen sind, und einer immer restriktiver werdenden Aufnahmepolitik der „klassischen“ Zielländer, v.a. im globalen Norden, hat sich zunehmend die Erkenntnis durchgesetzt, dass die bisherigen Instrumente des Schutzes von Flüchtlingen und Migrant/inn/en ${ }^{6}$ heute nicht mehr ausreichend sind (vgl. Yaghmaian 2016, p. 2; IOM 2017, pp. 2 f.; UNHCR 2016 a, b).

Seit einigen Jahren gibt es daher auf der internationalen Ebene verstärkt Bemühungen, ein neues Regelwerk zum besseren Schutz von Flüchtlingen und Migrant/inn/ en, insbesondere irregulären Migrant/inn/en, zu entwickeln. $\mathrm{Zu}$ nennen sind hier in erster Linie die „New York Declaration for Refugees and Migrants ${ }^{\text {“7 }}$, das „Vulnerability Screeing Tool“ des UNHCR ${ }^{8}$, der „International Dialogue on Migration“ des IOM ${ }^{9}$, der „Global Compact on Refugees“ der $\mathrm{UN}^{10}$, der „Global Compact for Safe, Orderly and

$6 \quad$ Z.B. The 1951 Refugee Convention, the 1969 Organization of African Unity (OAU) Convention Governing Specific Aspects of Refugee Problems in Africa, the International Human Rights Law, the International Labour Law etc.

7 Die „New York Declaration for Refugees and Migrants“ wurde am 19. September 2016 von der UN-Generalversammlung verabschiedet und drückt den politischen Willen der Mitgliedsstaaten aus, die Rechte von Flüchtlingen und Migrant/inn/en zu stärken (vgl. UNHCR 2016 a, b; UN-GeneralVERSAMMLUNG 2016). Dazu wurden zwei Rahmenvereinbarungen - der „Global Compact on Refugees“ sowie der „Global Global Compact for Safe, Orderly and Regional Migration“ - ausgearbeitet, die 2018 verabschiedet wurden (vgl. UNHCR o.J.; IOM o.J.; UNITED Nations Human Rights Council 2017; UN National Assembly 2018) (vgl. auch Atak et al. 2018, p. 1).

8 Das „Vulnerability Screening Tool“ wurde vom UNHCR gemeinsam mit der INTERnational Detention Coalition [IDC] und der OAK Foundation entwickelt. „Its purpose is to identify situations of vulnerability so as to inform a range of decisions around the person such as those related to the most appropriate placement and support options in the individual case. It aims to encourage early intervention, effective care of individuals in need, and partnerships with community services. Further, it aims to reduce the presumption of detention and to encourage a consideration of placement options starting with the least restriction on liberty and freedom of movement" (UNHCR et al. 2016, p. 1).

9 Der „International Dialogue on Migration“ wurde im Jahr 2017 durch die International Organization for Migration zur Vorbereitung des „Global Compact for Safe, Orderly and Regional Migration“ organisiert. Im Verlauf zweier Tagungen wurden verschiedene Facetten der Vulnerabilität von Migrant/inn/en, v.a. in Situationen der irregulären Migration, besprochen und Vorschläge zum besseren Schutz und zum Empowerment dieser Personengruppe entwickelt (vgl. IOM 2017). 10 Der „Global Compact on Refugees“ basiert auf der „New York Declaration for Refugees and Migrants“ und wurde 2018 der UN-Generalversammlung seitens des Hohen Flüchtlingskommissars der UN vorgelegt und am 17. Dezember 2018 mit den Stimmen von 181 Staaten offiziell angenommen. Er enthält u.a. weitreichende Vorschläge zur Identifikation und zum Schutz vulnerabler Personen, vor allem in ,großen Fluchtbewegungen“ (vgl. UNHCR o.J. und UN-Generalversammlung 2018). 
Regional Migration“ der UN ${ }^{11}$ und die „Regulations and Directives on the Common European Asylum System“ (CEAS) ${ }^{12}$.

Gemeinsam ist den genannten Dokumenten und Richtlinien der Verweis darauf, dass es heute eine Vielzahl von unterschiedlichen Motiven zur Migration gibt und viele Flüchtlinge und Migrant/inn/en, vor allem wenn sie in „großen Migrationsströmen“13 migrieren, sowohl vor dem Beginn wie auch während ihrer Migration und danach mit vielen ähnlichen Risiken und Problemen (z.B. Ausbeutung und Missbrauch durch Schlepper, Menschenhändler oder skrupellose Arbeitgeber) konfrontiert sind.

Um dem Rechnung zu tragen, wurden in den erwähnten UN-Dokumenten u.a. Leitlinien zum Monitoring sowie zum Schutz und zur Unterstützung von „Persons in precarious/vulnerable situations“ bzw. von „refugees with special protection needs“ erlassen, die allerdings keinen bindenden Charakter haben. ${ }^{14}$

Exemplarisch möchte ich in diesem Zusammenhang auf das „,Vulnerability Screening Tool“ von UNHCR, InTERnational Detention CoALition \& OAK Foundation (2016) Bezug nehmen. In diesem wird zunächst festgehalten, dass „... vulnerability is more inclusive than being at risk of persecution or refoulement” (ebenda, p. 1). Situationen der Vulnerabilität können aus einer Vielzahl von Gründen entstehen, z.B. durch

11 Der „Global Compact for Safe, Orderly and Regional Migration“ basiert ebenfalls auf der „New York Declaration for Refugee and Migrants“ des Jahres 2016. Er wurde am 19. Dezember 2018 von 164 Mitgliedstaaten auf der UN-Generalversammlung angenommen. Österreich hat den Compact nicht unterzeichnet (vgl. United Nations Human Rights Council 2017; UN NatiONAL ASSEMBLy 2018 und IOM o.J.).

12 Beim Common European Asylum System (CEAS) handelt es sich um „...A framework of agreed rules which establish common procedures for international protection and a uniform status for those, who are granted refugee status or subsidiary protection based on the full and inclusive application of the Geneva Refugee Convention and Protocol and which aims to ensure fair and humane treatment of applicants for international protection, to harmonise asylum systems in the EU and reduce the differences between Member States on the basis of binding legislation, as well as to strengthen practical cooperation between national asylum administrations and the external dimension of asylum" (vgl. https://ec.europa.eu/home-affairs/what-we-do/networks/european migration_network/glossary_search/common-european-asylum-system-ceas_en).

13 „Der Begriff, große Migrationsströme“ lässt sich an einer Reihe von Kriterien festmachen, darunter der Zahl der ankommenden Menschen, der wirtschaftliche, soziale und geografische Kontext, die Reaktionskapazität des Empfangsstaats und die Auswirkungen eines plötzlichen oder anhaltenden Menschenstroms. Unter den Begriff fallen beispielsweise nicht die regulären Migrantenströme von einem Land in ein anderes. ,Große Ströme ' können gemischte Ströme aus Flüchtlingen und Migranten umfassen, die aus unterschiedlichen Gründen, aber möglicherweise auf ähnlichen Routen unterwegs sind“"(UN-GENERALVERSAMMLUNG 2016).

14 Ein weiterer bereits früher verwendeter Terminus ist jener der ,extremely vulnerable individuals“ (EVI). Laut den UNHCR EVI-Richtlinien werden darunter Personen verstanden „... who may be in life threatening situation, unable to help themselves, lacking family and community support or suffering from physical or mental trauma“ (THE WORLD BANK \& UNHCR 2011, p. 22). „In practical terms, these categories encompass 11 types of EVI cases: single females, single parents, unaccompanied elderly, unaccompanied minors, physically disabled, mentally ill, chronically ill, poor families (5 or more dependents without any livelihood strategy), drug addicts, medical cases, and special cases“ (ebenda). 
„circumstances in a person's home country, during their journey, after arrival and in their experience of the asylum or migration system itself“ (ebenda, p. 2). ${ }^{15}$ Des Weiteren wird betont, dass diese „Situations of vulnerability are not fixed and will change over time with changing circumstances“ (ebenda). Die Vulnerabilität selbst „... is shaped by personal (internal) factors and environmental (external) factors. These factors can be multiple and intersect so as to entrench and exacerbate risks of harm“ (ebenda). Gleichzeitig wird im „Vulnerability Screeing Tool“ unterstrichen, dass „...[people] possess coping ability, resourcefulness, agency, and capacity to make positive contributions to society. Individuals possess both 'inner strengths' as well as the resources of their cultural and social connections: family, personal networks and community ties“ (ebenda).

Außerdem wird darauf verwiesen, dass eine genaue Feststellung der Vulnerabilität durch Expert/inn/en erforderlich ist, da „... for a range of reasons (including the individual's ability to communicate, shame, lack of trust, the competency of the questioner) individuals may not be clear about their situation, or be hesitant to disclose certain experiences immediately if at all“ (ebenda). Als besonders vulnerable Personengruppen nennt das „Screeing Tool“ „Refugees, asylum-seekers, trafficked persons, stateless persons, irregular migrants and other non-nationals without legal status often experience situations of vulnerability - they are often exposed to heightened risks of harm and require special care, support and protection“ (ebenda, p. 1).

Um ihre Vulnerabilität festzustellen wird ein personenzentrierter und holistischer Ansatz vorgeschlagen und auf eine Reihe sogenannter „Vulnerability Domains“ (z.B. Child, Sexual Orientation, Protection Needs etc.; vgl. Abb. 1) Bezug genommen (vgl. ebenda, p. 3)

Dabei wird davon ausgegangen, dass sich diese „vulnerability domains“ oftmals überlappen und dass ,While some people will be identified with several vulnerability factors, others will not be identified with pre-determined categories of vulnerability yet still be at serious risk of harm. Everyone’s circumstances are multifaceted and dynamic“" (UNHCR, InTERnational Detention CoALition \& OAK Foundation 2016, p. 3).

Neben den bereits genannten „vulnerability domains“ verweist das „Vulnerability Screening Tool“ aber auch darauf, dass es im Zuge der Identifikation von Vulnerabilitätsfaktoren noch weitere „domains“ gibt, die mit den persönlichen und sozialen Umständen eines Individuums zusammenhängen (UNHCR, INTERNATIONAL DETENTION CoAlition \& OAK Foundation, p. 21). Beispielsweise kann eine Person einer Minderheit angehören, die im Ankunftsland xenophober Gewalt ausgesetzt sein kann (vgl. ebenda) oder Männer und Buben, die in der Diskussion um die Vulnerabilität oft ausgeklammert sind, ,... can be at risk of violence, self-harm, and recruitment by violent groups“ (ebenda).

15 Situationen der Vulnerabilität kann es zudem auch nach der Deportation geben, wie z.B. neuere Studien zeigen, siehe Details unten. 
Abb. 1: Vulnerability Domains

\begin{tabular}{|c|c|}
\hline Child & $\begin{array}{l}\text { - Unaccompanied or separated child } \\
\text { - Child accompanied by parent/s, other family members or guardians }\end{array}$ \\
\hline $\begin{array}{l}\text { Sex, Gender, } \\
\text { Gender Identity, } \\
\text { Sexual Orientation }\end{array}$ & $\begin{array}{l}\text { Pregnant woman or girl, or nursing mother } \\
\text { - Sole or primary carer/s (of dependant child, elderly person or person with a disa- } \\
\text { bility) } \\
\text { - Woman at risk of sexual or gender-based violence, or adult or child experiencing } \\
\text { family violence, exploitation or abuse } \\
\text { - Person at risk of violence due to their sexual orientation and/or gender identity } \\
\text { (LGBTI: lesbian, gay, bisexual, transgender or intersex persons) }\end{array}$ \\
\hline $\begin{array}{l}\text { Health and Welfare } \\
\text { Concerns }\end{array}$ & $\begin{array}{l}\text { Physical and mental health } \\
\text { - Risk of suicide } \\
\text { - Eisability } \\
\text { - Elderly person } \\
\text { - Dubstance addiction } \\
\text { - Destitution }\end{array}$ \\
\hline Protection Needs & $\begin{array}{l}\text { Refugee and asylum-seeker } \\
\text { Survivor of torture and trauma } \\
\text { Survivor of sexual or gender-based violence or other violent crime } \\
\text { - Victim of trafficking in persons } \\
\text { - Stateless person }\end{array}$ \\
\hline Other & $\begin{array}{l}\text { The interviewer has an opportunity to identify vulnerability factors not captured } \\
\text { by the previous domains }\end{array}$ \\
\hline
\end{tabular}

Quelle: UnHCR, International Detention Coalition \& OAK Foundation 2016, p. 3.

Zusätzlich zur Festlegung von verschiedenen Formen der Vulnerabilität wird in einigen Dokumenten (z.B. dem Global Compact on Refugees) zudem der Begriff „Menschen mit besonderen Bedürfnissen/persons with special needs“ genannt. Dazu zählen „... Kinder, einschließlich derjenigen, die unbegleitet oder von ihren Familien getrennt sind, gefährdete Frauen, Überlebende von Folter, Trauma, Menschenhandel, sexueller und geschlechtsspezifischer Gewalt, sexueller Ausbeutung und sexuellem Missbrauch oder schädlichen Praktiken, Personen, die medizinische Hilfe benötigen, behindert sind oder nicht lesen und schreiben können, Heranwachsende und Jugendliche sowie ältere Menschen“ (UN-GeneralversammLung 2018, p. 13).

Auch im Common European Asylum System (CEAS) wird auf die Begriffe „vulnerable Personen“ und „persons with special needs“ Bezug genommen (vgl. AIDA \& ECRE 2019 sowie HruschKa \& Leboeuf 2019, pp. 2 f.). Laut Artikel 21 der Direktive 2013/33/EU inkludiert die Kategorie „vulnerable Personen“ „,.. minors, unaccompanied minors, disabled people, elderly people, pregnant women, single parents with minor children, victims of human trafficking, persons with serious illnesses, persons with mental disorders and persons who have been subjected to torture, rape or other serious forms of psychological, physical or sexual violence, such as victims of female genital mutilation“ (AIDA \& ECRE 2019, p. 4; vgl. auch p. 15). 
Des Weiteren geht das CEAS davon aus, dass „... some asylum seekers are more vulnerable than others due to their individual characteristics or circumstances, and therefore require more attention“ (AIDA \& ECRE 2019, p. 9). Diese Erkenntnis führte dazu, dass in den Verzeichnissen zum Monitoring des Schutzbedarfes zwei gesonderte Kategorien, nämlich „in need of procedural guarantees“ und „vulnerable/with special reception needs“ aufgelistet sind (ebenda, p. 15, vgl. nachstehende Abbildung).

\section{Abb. 2: Kategorien des Schutzbedarfs gemäß EU-Direktiven}

\begin{tabular}{|c|c|c|}
\hline \multicolumn{3}{|c|}{ Lists of protected categories in Directives 2013/32/EU and 2013/33/EU } \\
\hline Category / Factor & $\begin{array}{l}\text { "In need of procedural } \\
\text { guarantees" }\end{array}$ & $\begin{array}{l}\text { "Vulnerable" / "with special } \\
\text { reception needs" }\end{array}$ \\
\hline Children & $\sqrt{ }$ & $\sqrt{ }$ \\
\hline Unaccompanied children & $x$ & $\sqrt{ }$ \\
\hline Disability & $\sqrt{ }$ & $\sqrt{ }$ \\
\hline Elderly & $\sqrt{ }$ & $\sqrt{ }$ \\
\hline Gender & $\sqrt{ }$ & $\mathrm{x}$ \\
\hline Pregnancy & $\sqrt{ }$ & $\sqrt{ }$ \\
\hline Single parents with minor children & $x$ & $\sqrt{ }$ \\
\hline Human trafficking & $x$ & $\sqrt{ }$ \\
\hline Serious illness & $\sqrt{ }$ & $\sqrt{ }$ \\
\hline Mental disorders & $\sqrt{ }$ & $\sqrt{ }$ \\
\hline Torture & $\sqrt{ }$ & $\sqrt{ }$ \\
\hline Rape & $\sqrt{ }$ & $\sqrt{ }$ \\
\hline $\begin{array}{l}\text { Other serious forms of psychological, } \\
\text { physical or sexual violence }\end{array}$ & $\sqrt{ }$ & $\sqrt{ }$ \\
\hline Female genital mutilation & $x$ & $\downarrow$ \\
\hline Sexual orientation or gender identity & $\sqrt{ }$ & $x$ \\
\hline
\end{tabular}

Anzumerken ist in Zusammenhang mit dem CEAS, dass es trotz langjähriger Bemühungen bislang nicht gelungen ist, ein EU-weit einheitliches Asylverfahrenssystem zu entwickeln. Dies betrifft auch die genannten EU-Direktiven 2013/32/EU und 2013/33/EU. Hierbei bestehen sowohl auf nationalstaatlicher wie auf innerstaatlicher Ebene (z.B. in Österreich Unterschiede nach den Bundesländern) erhebliche Differenzen, was die Implementierung der Richtlinien in den jeweiligen Gesetzgebungen der einzelnen EU-Mitgliedsstaaten angeht (vgl. AIDA \& ECRE 2019, pp. 15 ff.).

Zudem scheint es beträchtliche Divergenzen in Bezug auf die Festlegung vulnerabler Gruppen zu geben, wie HruschKa \& Leboeuf (2019, p. 3) feststellen: „As highlighted in an ECRE report (2017, p. 16), asylum seekers with mental disorders, for example, are recognised as vulnerable under Polish and Italian national law, but not under French and Spanish law. Victims of human trafficking are considered vulnerable in the context of the asylum procedures in Belgium and Greece, but not in Ireland or 
Croatia. EU Member States seem to apply their own understanding of the concept of 'vulnerability'."16

Zusammenfassend kann bezüglich des Vulnerabilitätsbegriffs im operativen Flüchtlings- und Migrant/inn/enschutz festgehalten werden, dass, wie das IOM konstatiert „... there is a lack of clarity on what is meant by the term 'vulnerable migrant', and what protection and assistance might be afforded to such migrants. There is a need for clarity around this term and agreement on the best way forward in meeting the significant operational challenges of providing protection and assistance to significant numbers of migrants in need of such services“ (IOM 2017). ${ }^{17}$ Das IOM plädiert daher dafür ein besseres Verständnis der Begriffe ,vulnerable migrants“ und „migrants in a situation of vulnerability“ zu entwickeln.

Kritisiert wird insbesondere der bisherige Diskurs rund um vulnerable Personen, der sich meist ,...exclusively on those with legal definitions and specified protections (e.g. refugees, trafficked persons), or on an individual's membership in groups (e.g. women, children, people with disabilities)“ bezieht (IOM 2017).

Dieser Fokus ist nach Ansicht des IOM jedoch aus mehreren Gründen problematisch, denn: „This approach can obscure the fact that, within these groups, vulnerabilities vary significantly. ... Further, classifying individuals as vulnerable due to their membership in a particular group does not take into account the many factors that may protect an individual from exploitation or abuse, regardless of their membership in said group, and downplays the agency of individuals and their abilities to overcome vulnerability factors and achieve their migration goals“ (IOM 2017). Zudem trägt dieser Ansatz zu einem „Protection gap“ bei „..., as protection actors may be blind to the needs of those who are not members of a protected class of migrant or of a group deemed vulnerable“ (IOM 2017, p. 3). Um diesen „Protection Gap“ zu adressieren, plädiert das IOM für ein umfassenderes Verständnis der Vulnerabilität. „... one that does not focus solely on protected categories of migrants, or on a migrant's membership in a particular group, but instead complements these approaches with a more complete understanding of the factors that contributed to the individual migrant's or group of migrants' vulnerability, and the resources and capacities they themselves can mobilize to resist or recover from their vulnerability, and which would apply at any stage of the migration process and in any context" (ebenda).

Vulnerabilität sollte deshalb laut dem IOM wie folgt definiert werden: „Within the migration context, vulnerability can be defined as the diminished capacity of an individual or group to resist, cope with, or recover from violence, exploitation, abuse, and/ or violation(s) of their rights. It is determined by the presence, absence and interaction of factors or circumstances that increase the risk of and exposure to, or protect against, violence, exploitation, abuse and rights violations“ (IOM 2017, p. 7). Des Weiteren

$16 \quad$ Allgemein zum Problem der Zuordnung vulnerabler Gruppen vgl. auch CRAWLEY \& SKLEPARIS 2018, pp. 58 f.; HyNdMAn 2000; YAGHMAIAN 2016.

17 Darauf verweisen auch AIDA \& ECRE 2019, p. 15; HruschKa \& Leboeuf 2019, p. 2; ATAK et al. 2018 sowie YaghMaian 2016. 
verweist das IOM darauf, dass es verschiedene Faktoren gibt, welche die Vulnerabilität verstärken bzw. verringern, wobei die relevanten Faktoren für verschiedene Personengruppen sehr unterschiedlich sein können (ebenda). „For example, being female or transgender increases an individual's risk of sexual exploitation and abuse, while being in an irregular migration status increases vulnerability to labour exploitation“ (ebenda).

Bei der Entwicklung von Schutzmaßnahmen auf der nationalen, regionalen und globalen Ebene ist es daher essentiell zu berücksichtigen, „... to what individuals, families, communities or groups of migrants are vulnerable to in a specific context, without focusing exclusively on recognized categories of vulnerable migrants“ (IOM 2017, p. 7). Zudem sollten Staaten bei der Feststellung oder Voraussage der Vulnerabilität von Individuen, Haushalten oder Gruppen immer eine holistische Evaluierung der Risikound Schutzfaktoren sowie der zur Verfügung stehenden Ressourcen vornehmen. (vgl. ebenda). ,Further, responses should differ, distinguishing between the needs that require an immediate short-term response and those that require prevention and long-term consideration, and resources should be directed to meet both kinds of needs, without leaving aside structural and long-term problems“ (ebenda).

\section{Das Vulnerabilitätsparadigma in der Flucht- und Migrationsforschung}

Nach diesen Ausführungen zum Vulnerabilitätsbegriff im operativen Flüchtlingsund Migrant/inn/enschutz möchte ich im Folgenden einen kurzen Überblick über die Behandlung des Vulnerabilitätsparadigmas in den Rechts- und Sozialwissenschaften geben, wobei ich etwas genauer auf die sozialwissenschaftlichen Diskurse zum Thema Vulnerabilität von Flüchtlingen und Migrant/inn/en eingehen werde.

Gemeinsam ist beiden Disziplinen die Annahme, dass eine strikte Trennung zwischen Flüchtlingen und Migrant/inn/en heute angesichts der großen globalen Migrationsbewegungen, die häufig durch eine Kombination unterschiedlicher Ursachen (z.B. langjähriges Displacement oder mehrfache Flucht in Folge andauernder Gewalt, die ihrerseits zu Verarmung und Perspektivenlosigkeit führen) ${ }^{18}$ ausgelöst werden, nicht mehr sinnvoll erscheint. ${ }^{19}$ Zahlreiche Forscher/inn/en, v.a. in den Sozialwissenschaften (wie nachstehend näher ausgeführt werden wird), plädieren daher für die Anwendung eines umfassenden Vulnerabilitätsbegriffes. ${ }^{20}$

18 Ad. multiple Migrations- und Fluchtgründe vgl. FidDiAn-QASMiYeH et al. 2014; BrüCKNER et al. 2016; Crawley \& Skleparis 2018; Donini et al. 2016; Betts 2013, pp. 16-18; Castles 2013; KLEIST 2015, 2018; ZeTTER 2007.

19 Vgl. Monsutti 2008, 2018, pp. 450, 454; Grawert \& Mielke 2018; Aleinikoff 2017; Crawley \& Skleparis 2018; Hyndman 2000.

20 Auch in einigen rezenten UN-Dokumenten wird diesem Aspekt Rechnung getragen (vgl. Ausführungen oben; siehe z.B. IOM 2017, UNHCR 2016a, b, UN-GENERALVERSAMMLUNG 2016). 
Schwerpunktmäßig befassen sich viele rechtswissenschaftliche Studien mit der Analyse des Vulnerabilitätsbegriffes in verschiedenen Rechtsdokumenten und programmatischen Erklärungen internationaler Organisationen (z.B. der „New York Declaration for Refugees and Migrants“ der UN-Generalversammlung, dem „International Dialogue on Migration“ des IOM oder dem Common European Asylum System) sowie den Vulnerabilitätsdefinitionen international agierender NGOs (z.B. Médecins du Monde) (vgl. Atak et al. 2018; HruschKa \& Leboeuf 2019). Dabei geht es u.a. um die Frage, wer in den diversen Rechtsdokumenten etc. als vulnerabel gilt und welche Implikationen das Label „Vulnerabilität“ hat. Diesbezüglich vermerken HruschKa \& LeBoeuf (2019, p. 2), dass „The use of 'vulnerability' by researchers, policymakers and stakeholders to develop and implement the legal and policy framework on asylum and migration entails inclusionary and exclusionary effects." ${ }^{\text {"21 }}$

Ein illustratives Beispiel dafür ist die aktuelle Situation auf den griechischen Inseln (z.B. im völlig überfüllten Camp Moria auf Lesbos). Nur wer als besonders vulnerabel gilt, kann Moria Richtung griechisches Festland verlassen oder wird in besser ausgestatteten Einrichtungen auf der Insel untergebracht (vgl. Howden \& KodalaK 2018 und CANTAT 2018, p. 7). Dies führt dazu, dass „... migrants increasingly have to demonstrate extreme forms of suffering, such as advanced illnesses or intensive mental distress“ (CANTAT 2018, p. 7). Diese Notwendigkeit „,... to perform 'vulnerability' in order to leave the island of Lesvos ... place people in liminal places where demonstrating victimhood constitute the criteria upon which the decision of extending aid is based“" (ebenda, pp. 9 f.).

Andere rechtswissenschaftliche Studien untersuchen die tatsächliche Umsetzung von Schutzempfehlungen für vulnerable Gruppen (z.B. die Umsetzung des Common European Asylum System in den einzelnen Mitgliedstaaten der EU) (vgl. HruschKA \& Leboeuf 2019; AtaK et al. 2018). Beispielsweise vermerkt Pervou (2017), dass UMF (Unbegleitete Minderjährige Flüchtlinge), obwohl sie als besonders vulnerable Gruppe gelten, im Asylsystem Griechenlands nicht ausreichend geschützt sind.

Resümierend kritisieren zahlreiche Rechtswissenschaftler/innen die unklare Definition des Begriffs Vulnerabilität (vgl. Yaghmanian 2016; HruschKa \& Leboeuf 2019; AтAK et al. 2018) sowie die Art und Weise der Kategorisierung vulnerabler Gruppen. ${ }^{22}$ Außerdem monieren sie, dass einzelne Stakeholder (z.B. UNHCR, IOM, EU-Mitgliedstaaten und NGOs) sehr unterschiedliche Kriterien und Assessment-Strategien zur Festlegung von Vulnerabilität heranziehen. „Some prefer to define clear categories, for instance based on group membership, while others prefer to work with a blurred notion which allows for flexibility in their action“ (HruschKa \& Leboeuf 2019, p. 3).

Das Fehlen einer klaren, allgemein verbindlichen Definition von Vulnerabilität hat für die Betroffenen jedoch Konsequenzen, wie z.B. HruschKA und Leboeuf (2019, p. 4) ausführen: „In the EU, national legislators tend to have their own understanding

$21 \quad$ Vgl. auch NAwYN (2012, p. 57), die hier Bezug nimmt auf AgIER (2011).

22 Auch das IOM hat auf dieses Problem hingewiesen und daher einen alternativen Vorschlag zur Definition und Messung der Vulnerabilität vorgelegt. 
of what vulnerabilities are. This creates protection gaps: One vulnerable group may be protected by the asylum law of one European country and not recognized as such in a neighboring one. For stakeholders working on vulnerable groups, this implies that stakeholders define their own criteria." Daher plädierten Expert/inn/en auf einer von „Population Europe“ $2018^{23}$ organisierten Konferenz zum Thema „Vulnerabilität“ für ein systematischeres Verständnis der Vulnerabilität von Migrant/inn/en, Asylsuchenden und Geflüchteten. Dies ist erforderlich, um zu verhindern, dass Gerichte, Gemeinden, Zivilgesellschaft und andere Akteure ihr jeweils eigenes Verständnis von Vulnerabilität anwenden: „Therefore, there is a need for a better understanding of the evolution of vulnerabilities over time, in all phases of the migration experience. The absence of a clear definition of "vulnerable migrants" indicates a need for a more comprehensive analysis of vulnerability“ (ebenda).

Um dieses bessere Verständnis zu erreichen, könnten laut HruschKa \& LEBOEUF (2019, p. 4) insbesondere die Sozialwissenschaften, die ja seit langem vulnerable Bevölkerungen studieren, den Richter/inne/n, die mit diesen Gruppen arbeiten, wertvolle Einblicke liefern. Insgesamt wäre eine derartige interdisziplinäre Zusammenarbeit sehr hilfreich. „Interdisciplinary collaboration between social scientists and lawyers may help to counteract the lack of a definition of 'vulnerable migrants' and provide more comprehensive analyses of the vulnerabilities as they are lived and experienced by migrants, refugees, and asylum seekers, including the coping strategies and the way such strategies are adapted within and in response to the relevant legal framework, as well as of their evolution over time“ (ebenda, p. 3).

In der sozialwissenschaftlichen Forschung zum Vulnerabilitätsparadigma lassen sich mehrere Themenschwerpunkte festmachen. Zum einen befassen sich zahlreiche Forscher/innen - wie die Rechtswissenschaftler/innen - mit dem Begriff der Vulnerabilität selbst und untersuchen u.a. die Anwendung des Vulnerabilitätsparadigmas im operativen Flüchtlings- und Migrant/inn/enschutz (vgl. z.B. KRAUSE \& ScHMIDT 2019; HruschKa \& Leboeuf 2019; AtaK et al. 2018). Allerdings gehen viele Sozialwissenschaftler/innen von einem deutlich umfassenderen Verständnis des Begriffs aus. Vulnerabilität wird meist breiter definiert, mit sozialer, ökonomischer und politischer Exklusion in Beziehung gesetzt und verweist somit ganz allgemein auf Personen, die marginalisiert und ausgegrenzt werden (vgl. STEWART 2005, p. 499). Neben Asylwerber/ inne/n und Opfern des Menschenhandels werden daher auch abgelehnte Asylwerber/ innen und Personen, die nur den Status einer „Duldung“ besitzen (beides Gruppen, die als „Deportable“ gelten) sowie deportierte Migrant/inn/en und Flüchtlinge untersucht. ${ }^{24}$

${ }^{23}$ An dieser Konferenz nahmen sowohl Rechts- und Sozialwissenschaftler/innen wie auch Vertreter/innen internationaler Organisationen (z.B. UNHCR, Médecins sans Frontier, European Council of Refugees and Exiles etc.) teil. Bezüglich der Details vgl. HruschKa \& Leboeuf (2019, p. 2).

24 Vgl. Cabot 2014; Crawley et al. 2011; Stewart 2005; Gehrig 2004; Pervou 2017; De Genova 2002, 2013, 2019; De Genova \& Peutz 2010; Khosravi 2018a, b; Wyss 2019; Parla 2019; PiCOZZA 2017; MAJIDI 2018. 
Im Zusammenhang mit dieser breiter gefassten Gruppe vulnerabler Personen wird zudem den unterschiedlichen Phasen der Vulnerabilität und ihren jeweiligen Implikationen (z.B. Vulnerabilität während der Flucht, während des Asylverfahrens bzw. nach Ablehnung eines Asylantrages, nach Zuerkennung eines Asylstatus oder nach einer Deportation) besonderes Augenmerk geschenkt. Zahlreiche Studien befassen sich z.B. mit dem chronische Warten von Asylwerber/inne/ $\mathrm{n}^{25}$ und dessen Implikationen, den prekären Arbeits- und Lebensverhältnissen von illegalen Migrant/inn/en, abgelehnten Asylwerber/inne/n oder anderen als deportierbar geltenden Personen. ${ }^{26}$

Zum anderen zählen das Studium der Repräsentation von Geflüchteten und Migrant/inn/en als „vulnerablen Gruppen“ (z.B. ÇELIK-Rappas 2017; Hyndman \& Giles 2011; RaJaram 2002; MalKKi 1996; Sigona 2014) und das Labelling bzw. die Taxonomie unterschiedlicher Grade der Vulnerabilität (vgl. ZetTER 1991, 2007; Krause 2016; Howden \& KoDALAK 2018) zu einem weiteren Themenschwerpunkt sozialwissenschaftlicher Forschung (vgl. LoRENz 2018, p. 66).

Ein drittes Forschungsfeld steht in engem Konnex mit der Etablierung immer neuer Border-Regime und der sich, v.a. im globalen Norden, zunehmend verschärfenden Migrations- und Asylpolitik. Zahlreiche Sozialwissenschaftler/innen befassen sich mit den diversen Ausgrenzungsmechanismen von Flüchtlingen und Migrant/inn/ $\mathrm{en}^{27}$, die, wie einige Autor/inn/en (vgl. z.B. AtaK et al. 2018, pp. 4 f.; LABMAn 2012; HAN 2018; Lorey 2011, 2015; HAINMÜLLER et al. 2016; KHOSRAVI 2018a, 2019) konstatieren, als „Produktion von Vulnerabilität“ gewertet werden müssen. Auf diese beiden zuletzt genannten Forschungsfelder soll im Folgenden näher eingegangen werden.

In der (medialen) Darstellung von Geflüchteten lassen sich, wie LoRENZ (2018, p. 60) mit Bezugnahme auf Horst (2008) darlegt, zwei dominante Narrative ausmachen: Zum einen werden die Geflüchteten als „Gefahr“, als „Flut“" und „Bedrohung“ dargestellt, wobei dies insbesondere für junge, alleinstehende Männern gilt (vgl. ScHEIBELHOFER 2017, 2019 sowie Olivius 2016a, b). Zum anderen wird auf die Verletzlichkeit und Hilflosigkeit von Geflüchteten, und hier v.a. von Frauen und Kindern, verwiesen (vgl. MALKKi 1995a; CARPENTER 2006; LoRENZ 2018, pp. 61, 67 f.). ${ }^{28}$

25 Bendixsen \& ERIKSen 2018; BreKke 2004, 2010; Conlon 2011a, b; Griffiths 2014; Hyndman \& Giles 2011; JefFrey 2008; KhosRavi 2014, 2018c, 2019; Kohli \& KaukKo 2018; PASCuCCi 2015; Rotter 2010, 2016; MarbaCH et al. 2018; HainmüLler et al. 2016; LiLJA et al. 2019; Mountz et al. 2002; Stewart 2005; Brigden \& Mainwaring 2016.

26 CABot 2019; Crawley et al. 2011; Donini 2019; Golash Boza 2015; Goldring et al. 2009, 2011; GonZales 2015; GonZales et al. 2019; Griffiths et al. 2013; Hainmueller et al. 2016; Han 2018; KhosRavi 2010, 2018a, 2019; De Genova 2013; Lewis et al. 2015; LiLja et al. 2019; Marbach et al. 2018; Parla 2019; Polanco 2016; Standing 2014; Stewart 2005; Lyons 2017; CodDington et al. 2020.

27 Brigden \& Mainwaring 2016; Cabot 2014; Coddington et al. 2020; De Genova 2013, 2017; De Genova \& Peutz 2010; De Genova \& Roy 2020; ECRE 2017; Golash-Boza 2015; GonZaLes et al. 2019; Koshravi 2010, 2019.

28 Vor allem Bilder von Kindern (z.B. das Photo von Aylan Kurdi) dienen oft als Symbol für das „suffering of refugees“ (Pervou 2017, pp. 4, 6; vgl. auch Çelik-RaPPAS 2017). 
Insgesamt erfolgt, wie LORENZ bezüglich der Repräsentation von Geflüchteten, aber auch von anderen Konflikt- und Katastrophenopfern, v.a. im globalen Süden, feststellt, eine Art Pathologisierung bzw. Viktimisierung bei gleichzeitiger Essentialisierung und Exotisierung ihrer Vulnerabilität (vgl. LoRENZ 2018, pp. 70 ff.). ${ }^{29}$

Insbesondere Flüchtlinge werden, wie zahlreiche Autor/inn/en (vgl. BAINEs 2004; Betts \& Loescher 2011; CARPenter 2006; Turner 2010; Lorenz 2018, p. 67) aufgezeigt haben, häufig durch ihre vermeintliche und/oder reale Vulnerabilität charakterisiert. Sie erscheinen als „paradigmatisch vulnerable Personen“ (LoRENZ 2018, p. 67), als „prototypical face of the [humanitarian; Anm. Lorenz] emercency“ (CALHOUN 2010, p. 33; zitiert nach LoRENZ 2018, p. 61), als „pure victims in general“ (MALKKI 1996, p. 378) oder wie Newman (2003, p. 10) feststellt, als „the most vulnerable group“ (zitiert nach LoRENZ 2018, p. 67). Sigona (2014, p. 370) wiederum beschreibt die Lebensverhältnisse von Flüchtlingen als „pure victimhood and vulnerability“, während HoRsT (2008, p. 13) von „bare humanity and extreme vulnerability“ spricht (vgl. LoRENz 2018, pp. 61, 67). Gleichzeitig werden sie, und hier insbesondere Frauen und Kinder, nicht nur als vulnerabel im Sinne von „anfällig“ charakterisiert (vgl. ebenda, p. 79), sondern häufig auch als ,powerless victims“ (LuDwIG 2013, p. 5), „vulnerable victims“ (HorST 2008, p. 2) oder als „helpless victims [...] rendered speechless and without agency“ (RAJARAM 2002, p. 251). ${ }^{30}$

Diese Repräsentationen stellen jedoch, wie САВOт bezugnehmend auf FAssin (2007, p. 512; ONG 2003 und TICKTIN 2017) konstatiert, eine Konstruktion dar. Es sind die Praktiken der Hilfezuteilung, welche die „... figure (FASSIN 2007, p. 512) of the deserving aid recipient“ produzieren „... framing him or her as a victim in need of being protected or saved“ (САвот 2014, p. 111). Vielfach werden, wie LoRENZ (2018, pp. 61, 67) mit Verweis auf CARPENTER 2006, AgIER 2011 und TURner 2010 anmerkt „Binnenklassifizierungen und Abstufungen der Vulnerabilität“ vorgenommen um, wie es MALKKI (1996, p. 384) in ihrem Beitrag „Speechless Emissaries“ formulierte, „exemplary victims“ als die Vulnerabelsten unter den Vulnerablen zu identifizieren oder wie AGIER bezüglich der Kategorie „Flüchtlinge“ feststellt. „The category of 'refugee’ is itself broken down into distinct categories of vulnerability, which end up by establishing a hierarchy of misfortune“ (AGIER 2011, p. 213 in LoRENZ 2018, p. 67).

$\mathrm{Zu}$ den Vulnerabelsten unter den Vulnerablen zählen, „... allen voran Kinder, gefolgt von Alten, Kranken und Frauen“ (Lorenz 2018, p. 61; vgl. auch CARPENTER 2006). Die Klassifizierungen und Zuschreibungen orientieren sich hierbei, wie LoRENZ (2018, pp. 60 ff.) ausführt, vielfach an genderspezifischen Ordnungen (vgl. ebenda; Baines 2004; MALKKI 1995a; Olivius 2016a, b).

29 Dies gilt auch, obwohl von LoRENz nicht erwähnt, wie andere Autoren konstatieren, für die Darstellung illegaler Migrant/inn/en (vgl. z.B. De Genova 2013, p. 1191), der die illegalen Migrant/inn/en als ,pitiful and helpless (feminized) 'victims' of migrant 'smuggling'“ beschreibt. 30 Vgl. auch Turner 2010; Kibreab 1993; Lorenz 2018, p. 70. Dieser Ansatz des hilflosen Opfers wird heute von zahlreichen Autor/inn/en kritisiert, die darauf verweisen, dass Flüchtlinge und andere vulnerable Gruppen Agency und Resilience besitzen (ad Details siehe unten). 
Heute allerdings sind, wie das Beispiel der Flüchtlingslager auf den griechischen Inseln anschaulich illustriert, Geschlecht und Alter oder ein anderes Vulnerabilitätskriterium, kein hinreichender Grund mehr für eine Verlegung auf das griechische Festland. Diese Differenzierung in jene „... who 'deserve' to be released [Anm.: aus Camps wie Moria auf Lesbos] and those who don't" (ebenda), bewirkt, wie Howden \& KodalaK (2018, pp. 1 f.) ausführen, eine Art „vulnerability contest“ und „Lottery of Protection“.

Die Fokussierung auf die Vulnerabilität und „Deservingness“ ist zudem zutiefst problematisch. „Indeed, it links the access to certain rights not to political subjectivities but rather to one's position as a victim“ (CANTAT 2018, p. 7). Damit werden bestimmte „regimes of vulnerability and deservingness“ geschaffen, die wie CANTAT bezugnehmend auf MaLKKi 1996 und Chauvin \& GarcÉs-Mascareñas 2014 vermerkt, darauf hindeuten, dass ,mechanisms of disciplining and control [are] increasingly premised on the suppression of migrants’ political agency“ (CANTAT 2018, p. 7; vgl. auch HARRELLBoND 1999).

Gleichzeitig stellt die Konstruktion des hilflosen Flüchtlings, welcher der Unterstützung bedarf, weil er sich nicht selbst helfen kann, auch ein Legitimierungsinstrument für Interventionen dar (vgl. DE GENova 2013, p. 119; BANKoff 2001, p. 27; FASSIN 2010; AgIER 2011; LoREnz 2018, p. 75) und ermöglicht es, über die Akkumulation von Informationen über vulnerable Gruppen auch Kontrolle über diese auszuüben, wie dies die beiden nachfolgenden Zitate illustrieren: „Getting to know (or discursively framing) the Third World is also about getting to discipline and monitor it, to have a more manageable Other“ (KAPOOR 2008, p. 46) „Kontrollwissen und Hilfe gehören für Michael AgIER unweigerlich zusammen. „There is no care without control!“ (AGIER 2011, p. 4) (zitiert nach LoRENZ 2018, p. 75).

Neben der Pathologisierung und Viktimisierung von Geflüchteten als hilflosen Opfern ist die Repräsentation von Flüchtlingen laut LoRENZ (2018, pp. 71 ff.) zudem durch eine Essentialisierung und Exotisierung ihrer Vulnerabilität charakterisiert. Insbesondere Geflüchtete werden, wie bereits mehrfach betont wurde, umfassende ,inherent vulnerabilities“ (BAINes 2004, p. IIX; vgl. LoRENZ 2018, p. 72) zugeschrieben. Indem das Vulnerabilitätsparadigma als das konstituierende Element der Flüchtlingserfahrung postuliert wird, erfolgt gleichzeitig eine Essentialisierung und Generalisierung der Vulnerabilität von Geflüchteten (vgl. MALKKI 1995b; RAJARAM 2002). ${ }^{31}$

Diese Essentialisierung von Geflüchteten wird v.a. am Beispiel der Darstellung geflüchteter Frauen deutlich. Sie werden häufig als besonders vulnerable Gruppen und als „pure victims of backward patriacharies“ (OLIvius 2016a, p. 286) bzw. als „victims of backward cultures“ (ebenda, p. 282) oder als „victims of archaic men” (SCHEIBELHOFER 2019, p. 198) beschrieben. Im Gegensatz dazu werden männliche Flüchtlinge oft als „perpetators“ und als „victims of uncivilized masculinity“ (OLIVIUs 2016a, p. 283

31 Hier ist allerdings anzumerken, dass nicht alle Flüchtlinge vulnerabel sind. Vgl. hierzu die Ausführungen oben nach IOM sowie Krause \& Schmidt (2019, p. 1), die ebenfalls Kritik an dieser Essentialisierung üben. 
und 2016b) dargestellt. Mit diesem Verweis auf die „backward patriacharies“, deren Opfer die geflüchteten Frauen aus dem globalen Süden sind, wird laut LoRENZ auf den altbekannten kolonialen Topos „weiße Männer retten braune Frauen vor braunen Männern“ (vgl. SPIVAK 2008) Bezug genommen und die Geflüchteten als ,exotic vulnerable others“ (BAINES 2004, p. 36; vgl. PARPART 1995; Horst 2008) sowie als „unterlegene[n] und zivilisationsbedürftige[n] Andere[n] repräsentiert“ (REUTER \& VILLA 2010, p. 26, zitiert nach LoRENZ 2018, p. 73).

Diese binäre Darstellung geflüchteter Frauen als „vulnerable victims of backward patriarchies“ und der Männer, denen „in Anbetracht der „social structures and norms that have socialized them into this position“ (OLIVIUs 2016a, p. 283), sowohl ein Opfer- wie auch ein Täterstatus zugeschrieben wird, erfährt heute vielfach Kritik. (Olivius 2016 a, b). Auch geflüchtete Männer, insbesondere alleinstehende, junge Männer, können - obwohl sie gemeinhin nicht als vulnerabel gelten - von Vulnerabilität betroffen sein. ${ }^{32}$ Für sie gibt es wenige Unterstützungsprogramme (vgl. ScHeIBELHOFER 2019, pp. 198 f.; Olivius 2016a, p. 279; Scalettaris et al. 2019; Wyss 2018, p. 122). Ihnen wird Misstrauen entgegengebracht (SCheibelHofer 2019, p. 209; NeVE 2014, p. 47) und sie sind oftmals mit einem ,racial profiling“ konfrontiert (SCHEIBELHOFER 2019, p. 210).

Zahlreiche Autor/inn/en kritisieren mittlerweile diese Repräsentation und Essentialisierung von Geflüchteten und illegalen Migrant/inn/en als ,powerless and pure victims“ und betonen, dass diese nicht nur Opfer sind, die der Hilfe bedürfen, sondern dass sie auch Resilience and Agency besitzen (vgl. AgIER 2011; BAIRD 2014; BRIGDEN \& Mainwaring 2016; Krause \& Schmidt 2019; LAKRaA 2017; Harrell-Bond 1999). ${ }^{33}$ Vielmehr verweisen sie darauf, dass es heute vielfältige Mechanismen gibt, welche die Resilience und Agency von Flüchtlingen und Migrant/inn/en untergraben und damit deren Vulnerabilität perpetuieren würden. ${ }^{34}$ Sozialwissenschaftler/innen, die sich mit dieser Thematik befassen, vertreten die These, dass die Etablierung immer neuer Grenzsicherungsmechanismen und die gegenwärtig, v.a. im globalen Norden, zunehmend restriktiver werdende Flüchtlings-, Asyl- und Migrationspolitik sowie die wachsende Xenophobie zu einer verstärkten Vulnerabilisierung von Geflüchteten und Teilen der Migrant/inn/en führt und sprechen daher auch von einer „Produktion der Vulnerabilität““35 bzw. von der „Produktion eines Prekariats“. ${ }^{36}$

32 DAXner \& Nicola (2017, p. 20) bezeichnen alleinstehende Männer als vulnerabel, da es für sie weniger Programme als für Frauen, Jugendliche oder Familien gibt.

${ }_{33}$ UMF gelten allgemein als vulnerabel, aber sie zeigen, wie САвот (2014, p. 131) und LøNNING (2018) illustrieren, sehr viel Agency. Zum Beispiel versuchen sie „unsichtbar“ zu sein, um der Registrierung durch die Behörden in Griechenland zu entgehen (vgl. Pervou 2017, p. 11). Vgl. in diesem Zusammenhang auch die „strategic invisibilization“ (VILLEGAS 2010).

34 Vgl. Crawley et al. 2011; Goldring et al. 2009; De Genova 2013, v.a. p. 1180; Lorenz 2018; Stewart 2005; Lewis et al. 2015; Lilja et al. 2019; Han 2018; Cantat 2018, p. 7; PunZ 2019; v.a. pp. 61 f.; Suliman \& Weber 2019; LabMan 2012.

35 Vgl. AtaK et al. 2018; Han 2018; LiLja et al. 2019; Lewis et al. 2015; De Genova 2013.

36 LOREY 2011 spricht in diesem Zusammenhang von der governmentalen Prekarisierung; ad Details zum Prekariat siehe unten. 
Insbesondere die oft langen Asylverfahren, die geringen Anerkennungsquoten und der häufig nur mehr befristet gewährte Asylstatus von Personen mit subsidiärem Asylstatus oder humanitärem Bleiberecht sowie die Kürzungen von Unterstützungsprogrammen bewirken eine zunehmende Ausgrenzung und Marginalisierung und legen damit den Grundstein für eine sich verfestigende Vulnerabilität und Prekariatisierung. Davon sind v.a. Staatenlose, illegale Migrant/inn/en, abgelehnte Asylwerber/ innen, subsidiär Schutzberechtigte oder Personen mit einem humanitären Bleiberecht bzw. einem Duldungsstatus sowie Personen nach ihrer Deportation betroffen (vgl. Gehrig 2004; Lewis et al. 2015, Crawley et al. 2011; Stewart 2005; GonZales 2015; Gonzales et al. 2019; KHosRavi 2010, 2018a; Houte et al. 2016).

Auf Grund ihres Rechtsstatus ist ihr Handlungsspielraum stark eingeschränkt (vgl. Mulert 2019, p. 35; STEWART 2005, p. 500). Sie sind von zahlreichen Integrationsmaßnahmen (z.B. Deutschkursen und Berufsqualifizierungsprogrammen) ausgeschlossen. So verfügen sie über keinen legalen Zugang zum Arbeitsmarkt (MULERT 2019, p. 37 für die BRD; Punz 2019, pp. 61 ff. für Niederösterreich) und können in der Regel nur schwer auf dem regulären Immobilienmarkt eine Wohnung finden (vgl. KoHLBACHER 2019, pp. 143-170). Die Vulnerabilität von Asylwerber/inne/n steht daher - wie Stewart (2005, pp. 499 ff.) und CraWley et al. (2011) am Beispiel Großbritanniens illustrieren - in einem engen Konnex zu ihrer rechtlichen, sozialen und wirtschaftlichen Exklusion. ${ }^{37}$

Aber auch nach der Zuerkennung des Asylstatus sind zahlreiche Personen - trotz rechtlicher Voraussetzungen - häufig mit prekären Lebensverhältnissen konfrontiert (vgl. z.B. Lyons 2017 für UK). Bedingt durch die aufenthaltsrechtlichen Bestimmungen während des Asylverfahrens (z.B. kein Zugang zu Sprach- und Berufsqualifizierungskursen) sind sie meist schlecht auf die Zeit nach der Gewährung des Asylstatus vorbereitet. Viel wertvolle Zeit ist ungenützt verlorengegangen (vgl. Punz 2019, pp. 61 f.), um die seitens der staatlichen Behörden mit der Zuerkennung des Asylstatus nun erwartete rasche Integration in die Aufnahmegesellschaft und die Selbsterhaltungsfähigkeit durch eigene Arbeit, zu realisieren. ${ }^{38}$

Anerkannte Flüchtlinge können zwar theoretisch einer legalen Beschäftigung nachgehen und haben Zugang zu sozialen Benefits, aber „... face formidable structural barriers in accessing any employment. (...) Barriers include limited English language skills, non-recognition or no proof of qualifications awarded in other countries, discrimination and/or a reluctance by employers to take on workers who lack UK work 37 GeHrig (2004) erwähnt ähnliches für Personen, die lediglich den Status einer Duldung haben.

38 HainmüLler (et al. 2016, p. 1) illustrieren die Auswirkungen des langen Wartens auf die späteren Beschäftigungsmöglichkeiten: „We provide new causal evidence about how one central policy parameter, the length of time that refugees wait in limbo for a decision on their asylum claim, affects their subsequent economic integration. Exploiting exogenous variation in wait times and registry panel data covering refugees who applied in Switzerland between 1994 and 2004, we find that one additional year of waiting reduces the subsequent employment rate by 4 to 5 percentage points, a 16 to $23 \%$ drop compared to the average rate." 
experience or references- due to the lack of right to work while their asylum claim is processed (....)“ (LEwIS et al. 2015, p. 592). TÄUBIG (2009, p. 56) beschreibt diesen Umgang mit Asylwerber/inne/n als „organisierte Desintegration“.

Der gewährte Asylstatus und die damit verbundenen rechtlichen Voraussetzungen für eine nun theoretisch mögliche soziopolitische und ökonomische Inklusion ändert für zahlreiche Asylberechtigte kaum etwas an ihrer vulnerablen Situation oder an ihren prekären Lebensverhältnissen. Beispielsweise fällt es ihnen häufig schwer auf dem Arbeitsmarkt Fuß zu fassen, eine ihren Qualifikationen entsprechende Beschäftigung oder eine angemessene Wohnung zu finden.

Zahlreiche Sozialwissenschaftler/innen definieren Vulnerabilität daher als einen Prozess der Erzeugung von Ungleichheit und als ein Cluster von Nachteilen entlang sozialer Kategorien von Geschlecht, Alter, „race“, „ethnicity“ und Klasse (vgl. LoRENZ 2018, p. 63) bzw. als einen Zustand der soziopolitischen und ökonomischen Exklusion und Marginalisierung (STEWART 2005, p. 499) bei gleichzeitiger Inklusion in die neoliberale Wirtschaft, allerdings oft in prekären Arbeitsverhältnissen (DE GENOVA, 2013, p. 1180; Coddington et al. 2020; Baumann 2003; Goldring et al. 2009, 2011).

Beispielsweise vermerkt StEwART bezüglich der Asylsuchenden, dass diese „... face many spaces of exclusion. They are physically separated from the host society (e.g. due to housing areas) which prevents societal interaction. .... In addition, because asylum seekers have very little by way of financial resources, they are partially excluded from commercial spaces in cities. Asylum seekers are therefore paradoxically excluded from the cityscape within which they are forced to exist“ (STEwart 2005, p. 507). ${ }^{39}$

Auf die marginalisierte Position von Zuwander/ern/inne/n verweist auch KHosRAVI, wenn er konstatiert, dass „... undocumented migrants are not excluded but are excepted, they have not been thrown out, but neither are they considered participants. Undocumented migrants are included in society without being recognised as members“ (KHOSRAVI 2010, p. 95).

Andere Forscher/innen setzen die Produktion der Vulnerabilität von Flüchtlingen und Migrant/inn/en in enge Beziehung zur sozialen Desintegration (DöRRE 2014), zum Prekaritat (Goldring et al. 2009; LeWIS et al. 2015; LiLJA et al. 2019; GRIFFITHS et al. 2013) oder sogar zur „Destitution“ bzw. Mittellosigkeit (vgl. Crawley et al. 2011). Der Begriff „Prekariat“ bezieht sich hier meist auf jene ,... who find themselves subject to intermittent casual forms of labor“ (HAN 2018, p. 332), ${ }^{40}$ während der Begriff „Destitu-

39 Auf ähnliches verweist PASCUCCI 2015 in seiner Studie über junge irakische Flüchtlinge in Kairo, die zwar die dortigen Shoppingmalls frequentieren, aber nicht am allgemeinen Konsum teilhaben können.

40 Bezüglich Details zum Prekariat siehe Han 2018; Kasmir 2018; Standing 2014; Parla 2019. Bisweilen nehmen einzelne Autor/inn/en auch eine Differenzierung zwischen dem „Precariat“ und der „Precarity“ vor (vgl. z.B. Han 2018; p. 332). LoREY (2011) unterscheidet z.B. drei Dimensionen des Prekären: 1) das Prekärsein, 2) das Prekariat und 3) die governmentale Prekarisierung. Andere Autor/inn/en differenzieren zwischen Vulnerabilität und Prekariat (vgl. ATAK et al. 2018, p. 4 und LABMAN 2012). 
tion“ eine Situation umreißt, bei der die davon betroffenen Menschen nicht mehr in der Lage sind ihre grundlegenden Bedürfnisse (wie Nahrung, Wohnung, Gesundheitsversorgung etc.) zu befriedigen aber von institutioneller Unterstützung (z.B. durch staatliche Behörden) ausgeschlossen sind (CrAwley et al. 2011, p. 16). ${ }^{41}$ Gemeinsam ist diesen Ansätzen, dass die Produktion von Vulnerabilität, Prekariat und Destitution als eine „,bewusst herbeigeführte“ Strategie zur „Unterwerfung“ von Flüchtlingen und Migrant/ inn/en in die neoliberale globalisierte Wirtschaft verstanden wird (vgl. GoLDRING et al. 2009; Han 2018; Lyons 2017; Lilja et al. 2019; Suliman et al. 2010; CraWley et al. 2011; Dörre 2014; Lorey 2011; Bauman 2003; Coddington et al. 2020; Lewis et al. 2015). ${ }^{42}$

Durch eine zunehmend repressive Governmentalität, die eng mit der Schaffung von Unsicherheit und Angst verbunden ist (vgl. LEwIS et al. 2015, p. 592; LOREY 2011) sollen „willfährige“ Arbeitskräfte generiert werden, die bereit sind jede Form der Beschäftigung anzunehmen, um ihren Lebensunterhalt zu sichern. LOREY (2011) spricht in diesem Zusammenhang von einer „governmentalen Prekarisierung“ und davon, dass diese im Neoliberalismus zu einem Normalzustand geworden sei. ${ }^{43}$ Insbesondere illegale Migrant/inn/en und abgelehnte Asylwerber/innen sind davon betroffen (vgl. DE Genova 2013, v.a. p. 1181; CrAwLEY et al. 2011, pp. 16, 45 f. und 57). Für sie besteht nur die Möglichkeit der illegalen Arbeit, oftmals im informellen Sektor, wo sie mit „... low-skilled jobs, with low pay, long hours (or taking multiple jobs), poor working conditions, working weekends, and a constant fear of being raided by immigrant officials“ konfrontiert sind (CRAWLEY et al. 2011, p. 46). Sie werden nicht nur ökonomisch ausgebeutet, sondern sind auch rechtlich und sozial völlig marginalisiert. Für sie gelten weder arbeitsrechtliche Bestimmungen, noch haben sie Anspruch auf eine Gesundheitsversorgung oder andere soziale Benefits.

LEwIs et al. (2015, p. 593) bezeichnen diese Lebens- und Arbeitsverhältnisse als „Hyper-Prekariat“, welches durch die folgenden drei Manifestationen charakterisiert wird: „deportability, risk of bodily injury coupled with restricted access to healthcare, and transactional relationships.“ Anzumerken ist in Bezug auf das governmentale Prekariat und auf die Produktion der Vulnerabilität, dass davon nicht nur „illegale“

\footnotetext{
41 Ad Begriff Destitution: UNHCR, InTERnATIONAL Detention CoALition \& OAK FoundaTION (2016, p. 17) definieren Destitution wie folgt: „Destitution is a relative situation of vulnerability; however, not being able to meet basic needs can result in significant risks of harm such as: homelessness (including precarious and dangerous accommodation), inadequate nutrition, poor physical and mental condition, isolation, exploitation, abuse and high-risk taking behavior.”

Laut Crawley et al. (2011, p. 16) kann die Destitution/Mittellosigkeit während des gesamten Asylprozesses, von der Phase der Antragstellung bis zur Ablehnung eines Asylantrages, vorkommen. Besonders davon betroffen sind allerdings Langzeit-Asylwerber/innen, illegale Migrant/inn/en und abgelehnte Asylwerber/innen (Crawley et al. 2011, p. 16).

42 Goldring et al. (2009) haben diesen Prozess für temporäre Arbeitskräfte in Kanada beschrieben.

43 Eventuell sind vom governmentalen Prekariat im neoliberalen Kapitalismus alle betroffen (vgl. Савот 2019, p. 753; Han 2018, pp. 332 f.).
} 
Migrant/inn/en und abgelehnte Asylwerber/innen, sondern auch zahlreiche Asylberechtigte betroffen sind. Sie werden seitens der Behörden, die nach der Asylgewährung ihre möglichst rasche Selbsterhaltung durch eigen Arbeit einfordern, oft in prekäre Arbeitsund Lebensverhältnisse gedrängt. ${ }^{44}$

Um diesen Forderungen gerecht zu werden, verzichten viele auf zusätzliche Ausbildungsmöglichkeiten, die ihre Beschäftigungschancen erhöhen würden (MuLERT 2019), und ergreifen stattdessen die erstbeste Gelegenheit, um möglichst rasch Geld zu verdienen. Aufgrund der oben bereits angeführten „strukturellen Barrieren“ (vgl. LEwIS et al. 2015, p. 592; TÄUBig 2009, p. 56) während des Asylverfahrens bleibt ihnen oft nur die Option, als ungelernte Arbeitskräfte in als prekär geltenden Wirtschaftssektoren, wie z.B. der Gastronomie, dem Tourismus, Reinigungs- oder Baugewerbe, zu arbeiten. Oftmals stellen sie, ähnlich wie die Arbeitsmigrant/inn/en der 1960er und 1970er Jahre eine Art „Reservearmee“ auf dem Arbeitsmarkt dar (MulERT 2019, p. 35) oder wie CAntat (2018, p. 13) in Bezug auf junge Flüchtlinge formuliert „... the refugees of today are the proletariat of tomorrow“.

In den Sozialwissenschaften wird die Produktion der Vulnerabilität und des Hyperprekariats von Migrant/inn/en und Flüchtlingen zudem häufig mit der ,,sozialen Visibilität“ bzw. Invisibilität dieser Personengruppe in Verbindung gebracht (vgl. LAKRAA 2017; Baird 2014; Battistella 2017; ČAPO 2015; Villegas 2010; BorRen 2008). ${ }^{45}$ $\mathrm{Zu}$ jenen, die besonders davon betroffen sind, zählen Opfer des Menschenhandels, der irregulären und erzwungenen Migration, Hausangestellte und unbegleitete Minderjährige (BATTISTElla 2017) sowie staatenlose Flüchtlinge (Borren 2008) und abgelehnte Asylwerber/innen (Crawley et al. 2011).

Untersucht wird beispielsweise wie die Visibilität bzw. Invisibilität sowohl von Migrant/inn/en und Flüchtlingen wie auch von der Politik und den Behörden als strategisches Mittel eingesetzt wird (vgl. BorRen 2008; ČAPO 2015; VILLEGAS 2010; BAIRD 2014; LAKRAa 2017). In Anlehnung an MALKKI (1995), ${ }^{46}$ sprechen StEWART (2005) und VILLEGAS (2010) von einer „strategy of invisibility“, „strategy of visibility“ bzw. „Strategie der Invisibilisierung/Visibilisierung“.

In zahlreichen Studien (vgl. Stewart 2005, p. 207; Pervou 2017, p. 11; BaIrD 2014, pp. 3, 5 ff; VILLEGAS 2010) wird darauf verwiesen, dass beide Strategien einander nicht ausschließen und sowohl von Flüchtlingen und Migrant/inn/en als auch von Politik und Behörden genutzt werden. Um sich vor polizeilichen Kontrollen oder fremdenfeindlichen Übergriffen zu schützen, meiden viele Flüchtlinge und Migrant/inn/en öffentliche Orte und versuchen möglichst unsichtbar zu sein. Umgekehrt können sie

${ }_{44}$ Einen anderen Grund stellen die Remittance-Forderungen ihrer Familien im Herkunftsland dar.

45 Bislang existieren nur wenige Studien zur Visibilität bzw. Invisibilität von vulnerablen Gruppen, siehe v.a. LAKRAa 2017 ad Flüchtlinge; BaIRD (2014, p. 3) zu undokumentierten Migrant/inn/en und ihrer sozialen Visibilität.

46 MalKKI (1995a) hat diese „Strategy of Invisibility“ von Flüchtlingen als eine der ersten in einem Flüchtlingslager in Kenia beschrieben. 
aber durch eine erhöhte Visibilität (z.B. Hungerstreik, Protestmarsch, direkte Kontakte zu Medienvertreter/inne/n und Forscher/inne/n) versuchen, auf ihre prekäre Situation aufmerksam zu machen (ČAPO 2015, pp. 400-402; LAKRAA 2017; ANSEMS DE VRIES 2016; vgl. z.B. das Refugee Protest Camp Vienna, siehe AtaÇ 2016 und Mokre 2015).

Ähnliches gilt, wie ČAPO (2015) in Bezug auf die kurze Unterbringung von Flüchtlingen in Kroatien ausführt, für die Visibilisierungs-/Invisibilisierungsstrategien der Behörden. Um Proteste der lokalen Bevölkerung gegen die Anwesenheit von Flüchtlingen hintanzuhalten, werden diese in abgelegenen Gebieten untergebracht oder in ihrer Bewegungsfreiheit stark eingeschränkt. ${ }^{47}$ „,While the column was being formed, the refugees were not allowed to leave the buses, wait outside or stroll around the village. The system of monitoring was designed so that the refugees would be completely out of the way of the local population" (ebenda, p. 398). Es geht hierbei, wie sowohl CAPO (2015) als auch Pervou (2017, p. 11) und LAKRAA (2017) ausführen, darum, dass die Flüchtlinge für die lokale Bevölkerung möglichst unsichtbar bleiben.

Gleiches gilt für Arbeitsmigrant/inn/en, die, wie DonINI (2019, p. 15) für Qatar darlegt, möglichst ,invisible“ bleiben sollen. „The 'camps' where migrant workers live are conveniently out of sight. Workers are transported by bus to the spatially segregated areas where they live and where they become invisible ..., even on their days off they are not allowed to mingle in the parks with the Qatari locals. They must meet to chat, smoke, drink and exchange stories of aspirations and exploitation under dusty underpasses, in run-down squares where the sheer number of migrants forces their toleration by the authorities ....“ (Donini 2019, p. 15).

Allerdings nutzen auch Politik und Behörden die Strategie der Visibilisierung. Durch den permanenten Verweis auf die „Gefahren“, die von Asylwerber/inne/n und Migrant/inn/en aus „fremden Kulturen“ ausgehen (z.B. auf Plakaten und in Reden rechter Politiker/innen), wird eine erhöhte Aufmerksamkeit für ausländerfeindliche Themen erzeugt und damit eine verstärkte Visibilität dieser Gruppe im öffentlichen Diskurs geschaffen, um damit die eigenen politischen Ziele zu befördern (vgl. z.B. die bei SCHEIBELHOFER 2017 angeführten Beispiele).

Bisweilen kommt es auch zu einer Verschränkung beider Strategien, wie LAKRAA (2017) am Beispiel der medienwirksamen Zerstörung des „Jungles von Calais“ ausführt. Durch diese Aktion der französischen Behörden sollten die dort lebenden Flüchtlinge unsichtbar gemacht werden. „Ironically, the intention of the state to maintain the invisibility of refugees backfires and in fact makes them all the more visible“ (ebenda, p. 4).

Einige Sozialwissenschaftler/innen, die sich mit vulnerablen Gruppen, insbesondere „illegalen“ Migrant/inn/en und abgelehnten Asylwerber/inne/n und deren prekären Lebensumständen befassen, untersuchen in diesem Zusammenhang auch die Mobilität/ Immobilität dieser Gruppen. Im Unterschied zu anderen Studien rund um die Mobilität/Immobilität von Migrant/inn/en und Flüchtlingen, die meist die oft fragmen-

47 Vgl. in diesem Zusammenhang u.a. das ehemalige Asylwerber/innenquartier auf der Saualm in Kärnten. 
tierten Migrationsbewegungen vom Herkunftsland zum Zielort untersuchen und darauf verweisen, dass zwischen der Mobilität und Immobilität ein Zusammenhang besteht, dass Migrant/inn/en und Flüchtlinge diese Phasen der Immobilität in Kauf nehmen, um dann weiterreisen zu können (vgl. Brigden \& MAINwARING 2016, p. 428 und Wyss 2019), liegt der Hauptfokus hier nicht auf der Migration selbst, sondern auf den Situationen, mit denen Migrant/inn/en und Flüchtlinge nach ihrer Ankunft am Zielort konfrontiert sind. Dabei werden die Wechselwirkungen zwischen dem prekären rechtlichen und ökonomischen Status besonders in den Blick genommen. So verknüpft LAKRAA (2017) die Mobilität/Immobilität vulnerabler Gruppen mit deren sozialer Visibilität/Invisibilität. Um unsichtbar für die Behörden zu bleiben, sind viele gezwungen ihren Wohnort und Job häufig zu wechseln (vgl. ebenda). Diese hohe Mobilität erlaubt es ihnen jedoch selten irgendwo Fuß zu fassen, Kontakte zu knüpfen und so soziales Kapital aufzubauen. Sie bleiben in ihrer prekären Lage „gefangen“ (ebenda) und werden marginalisiert (BAIRD 2014, p. 6).

\section{Zusammenfassung}

Im vorliegenden Beitrag wurde versucht, einen Überblick über die Verwendung des Vulnerabilitätsparadigmas zu geben. Ursprünglich in der Katastrophenforschung entwickelt, fand dieses mittlerweile Eingang in eine Vielzahl unterschiedlicher Disziplinen, darunter auch in die Fluchtforschung.

Es konnte dargelegt werden, dass sich der Begriff der Vulnerabilität auf sehr verschiedene Dimensionen bezieht. Einerseits ist damit ganz allgemein der prekäre und sensible Status von Menschen auf der Flucht, die Schutz suchen, gemeint. Andererseits wird das Konzept der Vulnerabilität als ein Werkzeug gesehen, um besonders vulnerable Gruppen auszumachen (z.B. unbegleitete minderjährige Flüchtlinge, Frauen, Schwangere, Opfer von Folter, FGM etc.) und ihnen speziellen Schutz zu bieten. Obwohl der Begriff im operativen Flüchtlingsschutz omnipräsent ist, besteht bislang kein Konsens darüber, wer genau als vulnerabel gelten soll.

In der Fluchtforschung selbst wurde und wird Vulnerabilität unter zwei Gesichtspunkten untersucht: Zum einen geht es um die Repräsentation von Geflüchteten im globalen Norden und Süden. Sie werden als besonders vulnerable Personen dargestellt, die des Schutzes und der internationalen humanitären Hilfe bedürfen, wobei u.a. eine Taxonomie der Vulnerabilität vorgenommen wird. Während sich ein Großteil der älteren Arbeiten mit diesen Aspekten befasste, kritisieren neuere Arbeiten diese Repräsentation der ,pure victimhood“, der „,bare humanity and extreme vulnerability“ der Geflüchteten. Sie verweisen vielmehr darauf, dass Geflüchtete nicht nur Opfer sind, die Hilfe benötigen, sondern dass sie auch Agency aufweisen, die jedoch häufig durch legistische Rahmenbedingungen stark eingeschränkt wird. 
Zum anderen untersucht die rezente Fluchtforschung die „Produktion von Vulnerabilität“" von Geflüchteten. Dieser Ansatz geht davon aus, dass die zunehmend, v.a. im globalen Norden, immer restriktiver werdende Flüchtlings- und Asylpolitik sowie die wachsende Xenophobie zu einer verstärkten Vulnerabilisierung von Geflüchteten führt. Vulnerabilität wird hier als ein Prozess der Erzeugung von Ungleichheit und als „Cluster von Nachteilen“ entlang sozialer Kategorien von Geschlecht, Alter, Rasse/Ethnie und Klasse definiert und in enge Beziehung zum Prekariat gesetzt. Letzteres betrachten die Vertreter/innen dieses Ansatzes als ein Ergebnis der rezenten neoliberalen Wirtschaftsordnung und einer zunehmend repressiveren Governmentalität.

Einzelne Autor/inn/en sprechen sogar von einem „Hyperprekariat“ und beziehen sich dabei v.a. auf rezent zugewanderte Migrant/inn/en und Geflüchtete, die vielfach nur einen sehr begrenzten und zeitlich befristeten Zugang zum Arbeits- und Wohnungsmarkt haben, oft in illegale Beschäftigungsverhältnisse gedrängt werden und darüber hinaus häufig rechtlich und sozial marginalisiert sind. Die Vulnerabilität und das Hyperprekariat der betroffenen Migrant/inn/en und Geflüchteten werden zudem auch häufig mit der „sozialen Invisibilität“ und dem Themenkomplex Mobilisierung/Immobilisierung in Verbindung gebracht.

\section{Bibliographie}

Agier M. (2011), Managing the Undesirables: Refugee Camps and Humanitarian Government. Cambridge: Polity Press.

AIDA (= Asylum Information Data Base) \& ECRE (European Council on Refugees and Exiles) (2019), The concept of vulnerability in European Asylum procedures. Brussels, 31-08-2017. $<\mathrm{http}$ ://www.asylumineurope.org/sites/default/files/shadow-reports/aida_vulnerability_in asylum_procedures.pdf $>$ [Zugriff: 28-02-2020].

AleiniKoff A.T. (2017), Toward a Global System of Human Mobility: Three Thoughts. American Journal of International Law, 111, pp. 24-28. <DOI:10.1017/aju.2017.8.>.

ANSEMS DE VRIES L. (2016), Politics of (in)visibility: Governance-resistance and the constitution of refugee subjectivities in Malaysia. Review of International Studies, 42 (5), pp. 876-894. <DOI: https://doi.org/10.1017/S0260210516000103>.

Askola H. (2010), 'Illegal Migrants`, Gender and Vulnerability: The Case of the EU's Returns Directive. Feminist Legal Studies, 18, pp. 159-178.

ATAÇ I. (2016), 'Refugee Protest Camp Vienna': making citizens through locations of the protest movement. Citizenship Studies, 20 (5), pp. 629-646.

AtAK I., NAKachen D., Guild E. \& CRÉPEAu F. (2018), "Migrants in vulnerable situations" and the Global Compact for Safe Orderly and Regular Migration. Legal Studies Research Paper, 273, Queen Mary University of London. <https://papers.ssrn.com/sol3/papers. cfm?abstract_id=3124392> [Zugriff: 27-01-2020].

BAINes E.K. (2004), Vulnerable Bodies. Gender, the UN and the Global Refugee Crisis. Aldershot, Hants; Burlington, VT, Ashgate. 
BAIRD Th. (2014), The more you look the less you see. Visibility and invisibility of Sudanese migrants in Athens, Greece. Nordic Journal of Migration Research, 4 (1), pp. 3-10. <DOI: 10.2478/njmr-2013-0008>.

BANKofF G. (2001), Rendering the World Unsafe. >Vulnerability < as Western Discourse. Disasters, 25 (1), pp. 19-35.

BAnkoff G. (2007), Comparing Vulnerabilities. Toward Charting an Historical Trajectory of Disasters. Historical Social Research, 21 (3), pp. 103-114.

Battistella G. (2017), From Invisibility to Recognition: Reflections on the Conditions of Migrants in Our Society. Keynote address delivered at Exodus V [1]: August 31 - September 3, 2017, Singapore. <DOI: 10.14240/cmsesy 103117>; <https://cmsny.org/publications/invisibilityrecognition/> [Zugriff: 20-01-2020].

Bauman Z. (2003), Wasted Lives. Modernity and its Outcasts. Cambridge: Polity.

Bendixsen S. \& ERIKSEN Th. H. (2018), Time and the Other: Waiting and Hope among Irregular Migrants. In: JANEJA M.K. \& BandaK A. (eds.), Ethnographies of Waiting. Doubt, Hope and Uncertainty. London, Oxford etc., Bloomsbury, pp. 87-112.

BetTs A., (2013), Survival Migration: Failed Governance and the Crisis of Displacement. Ithaca: Cornell University Press.

Betts A. \& Loescher G. (2011), Refugees in International Relations. In: BetTs A. \& Loescher G. (eds.): Refugees in International Relations. Oxford, New York: Oxford University Press, pp. 1-27.

BorRen M. (2008), Towards an Arendtian politics of in/ visibility: on stateless refugees and undocumented aliens. Ethical Perspectives, Journal of the European Ethics Network, 15 (2), pp. 213- 237.

BREKKe J.-P. (2004), While we are waiting. Uncertainty and empowerment among asylum-seekers in Sweden. Oslo, Institute for Social Research, Report (10).

BrekKe J.-P. (2010), 'Life on Hold: Tue Impact of Time on Young Asylum Seekers Waiting for a Decision'. Diskurs Kindheits- und Jugendforschung 5 (2), pp. 159-167.

Brigden N. \& MaInwaring C. (2016), Matryoshka Journeys: Im/mobility During Migration. Geopolitics, 21 (2), pp. 407-434. <DOI: 10.1080/14650045.2015.1122592>.

Brown K., Ecclestone K. \& Emmel N. (2017), The Many Faces of Vulnerability. Social Policy \& Society, 16 (3), pp. 497-510. <https://www-cambridge-org.uaccess.univie.ac.at/core/ journals/social-policy-and-society/article/many-faces-of-vulnerability/2E9B5C9E2B5A6F 8253D6CD48E0FD0B08>.

Brücker H., Fendel T., Kunert A., Mangold U., Siegert M. \& Schupp J. (2016), Geflüchtete Menschen in Deutschland. Warum sie kommen, was sie mitbringen und welche Erfahrungen sie machen. IAB-Kurzbericht 15. Nürnberg, Bertelsmann.

Савот H. (2014), On the Doorstep of Europe: Asylum and Citizenship in Greece. Philadelphia, University of Pennsylvania Press.

Савот H. (2019), The European Refugee Crisis and Humanitarian Citizenship in Greece. Ethnos, 84 (5), pp. 747-771.

Calhoun C. (2010), The Idea of Emergency. Humanitarian Action and Global (Dis)Order. In: Fassin D. \& Pandolfi M. (eds.), Contemporary States of Emergency. The Politics of Military and Humanitarian Interventions. New York, NY, Zone Books, pp. 29-58.

Cantat C. (2018), The Politics of refugee solidarity in Greece: Border identities and political mobilization. CPS Working Papers, 2018/1. Budapest, Central European University Center for Policy Studies. 
ČAPO J. (2015), The Security-scape and the (In)Visibility of Refugees: Managing Refugee Flow through Croatia. Migracijske i etničke teme, 31 (3), pp. 387-406.

CARPEnTER R.Ch. (2006), Innocent Women and Children. Gender, Norms and the Protection of Civilians. Abingdon/UK and New York, Routledge.

Castles St. (2013), The Forces Driving Global Migration. Journal of Intercultural Studies, 34 (2), pp. 22-140.

ÇElik-Rappas I.A. (2017), Refugees as Innocent Bodies, Directors as Political Activists: Humanitarianism and Compassion in European Cinema. Revista Latinaamericana de Estudios sobre Cuerpos, Emociones y Sociedad, 23 (9), pp. 81-89.

Chambers R. (ed.) (1989), Vulnerability: How the Poor Cope. IDS Bulletin, 20 (2), pp. 1-7.

Chambers R. (2006), Vulnerability, Coping and Policy. Editorial Introduction: IDC Bulletin, 37 (4), pp. 33-40. <https://doi.org/10.1111/j.1759-5436.2006.tb00284.x>.

Chauvin S. \& Garcés-Mascareñas B. (2014), Becoming Less Illegal: Deservingness Frames and Undocumented Migrant Incorporation. Sociology Compass, 8 (4), pp. 422-432.

Clark I. (2013), The Vulnernable in International Society. Oxford: Oxford University Press.

Coddington K., Conlon D. \& Martin L.L. (2020), Destitution Economies: Circuits of Value in Asylum, Refugee, and Migration Control. Annals of the American Association of Geographers. <DOI: 10.1080/24694452.2020.1715196>.

Conlon D. (2011a), Waiting: feminist perspectives on the spacings/timings of migrant (im) mobility. Gender, Place and Culture, A Journal of Feminist Geography, 18 (3), pp. 353360.

Conlon D. (2011b), A Fractured Mosaic: Encounters with the Everyday amongst Refugee and Asylum Seeker Women. Population, Space, and Place, 17, pp. 714-726.

Crawley H. \& Skleparis D. (2018), Refugees, migrants, neither, both: categorical fetishism and the politics of bounding in Europe's 'migration crisis'. Journal of Ethnic and Migration Studies, 44 (1), pp. 48-64.

Crawley H., Hemmings J. \& Price N. (2011), Coping with Destitution. Survival and livelihood strategies of refused asylum seekers living in the UK. Centre for Migration Policy Research (CMPR), Swansea University.

CutTer S.L. (2003), The Vulnerability of Science and the Science of Vulnerability. Annals of the Association of American Geographers, 93 (1), pp. 1-12.

DaVid F., Byrant K. \& LaRsen J.J. (2019), Migrants and Their Vulnerability to Human Trafficking, Modern Slavery and Forced Labour. International Organization for Migration, Geneva.

DaXner M. \& Nicola S.-L. (2017), Mapping of and Report on the Afghan Diaspora in Germany, Centre for International Migration and Development. <https://www.cimonline.de/static/ media/cim2018-en-diaspora-afghanistan.pdf> [Zugriff: 02-11-2018].

De Genova N. (2002), Migrant 'Illegality'and Deportability in Everyday Life. Annual Review of Anthropology, 3, pp. 419-447.

De Genova N. (2013), Spectacles of migrant "illegality": the scene of exclusion, the obscene of inclusion. Ethnic and Racial Studies, 36 (7), pp. 1180-1198. <DOI: 10.1080/01419870.2013.783710>.

De Genova N. (2019), Detention, Deportation, and Waiting: Toward a Theory of Migrant Detainability. Gender a Výzkum, 20 (1), pp. 92-104. <DOI: 10.13060/25706578.2019.2 $0.1 .464>$. 
De Genova N. \& Peutz N. (eds.) (2010), The Deportation Regime. Sovereignty, Space, and the Freedom of Movement. Durham, N.C.M, Duke University Press.

De Genova N. \& Roy A. (2020), Practices of Illegalisation. Antipode, 52 (2), pp. 352-364.

DöRRE K. (2014), Precarity and Social Disintegration: A Relational Concept. JEP (Journal für Entwicklungspolitik), XXX (4), pp. 69-89. <https://doi.org/10.20446/JEP-2414-3197-304-69>.

Donini A. (2019), Social Suffering and Structural Violence: Nepali Workers in Qatar. International Development Policy/ Revue international de politique de développement, 11, pp. 1-19.

Donini A., Monsutti A. \& Scalettaris G. (2016), Afghans on the Move: Seeking Protection and Refuge in Europe. "In this journey I died several times; In Afghanistan you only die once”, Global Migration Research Paper, 17, Global Migration Centre, The Graduate Institute Geneva.

ECRE (2017), Vulnerability in European asylum procedures: New AIDA comparative report. <https:// www.ecre.org/vulnerability-in-european-asylum-procedures-new-aida-compara tive-report/> [Zugriff: 27-01-2020].

FAssin D. (2007), Humanitarianism as a Politics of Life. Public Culture, 19, pp. 499-520.

FAssin D. (2010), Heart of Humaneness. The Moral Economy of Humanitarian Intervention In: Fassin D. \& Pandolfi M. (eds.), Contemporary States of Emergency. The Politics of Military and Humanitarian Interventions. New York, New York, Zone Books, pp. 269-293.

Fiddian-QAsmiyeh E., Loescher G., Long K. \& Sigona N. (2014), Introduction: Refugee and Forced Migration Studies in Transition. In: Fiddian-QASMiYeh E., Loescher G., Long K. \& Sigona N. (eds.), The Oxford Handbook of Refugee and Forced Migration Studies. Oxford etc., Oxford University Press, pp.1-14.

GeHRIG T. (2004), The Afghan Experience of Asylum in Germany: Towards an Anthropology of Legal Categories. Tsantsa, Revue de la Society Suisse d'Ethnologie, 9, pp. 72-80.

Golash-Boza T.M. (2015), Deported: Immigrant Policing, Disposable Labor and Global Capitalism. New York, New York University Press.

Goldring L., Berinstein C. \& Bernhard J.K. (2009), Institutionalizing precarious migratory status in Canada. Citizenship Studies, 13 (3), pp. 239-265.

Goldring L. \& Landolt P. (2011), Caught in the Work-Citizenship Matrix: the Lasting Effects of Precarious Legal Status on Work for Toronto Immigrants. Globalizations, 8 (3), pp. 325341. <DOI: 10.1080/14747731.2011.576850>.

Gonzales R.G. (2015), Lives in Limbo: Undocumented and Coming of Age in America. Oakland, University of California Press.

Gonzales R.G., Sigona N., Franco M.C. \& Papoutsi A. (2019), Undocumented Migration: Borders, Immigration Enforcement, and Belonging. Cambridge/UK and Medford/MA, Polity Press.

Grawert E. \& Mielke K. (2018), Coping with protracted displacement. How Afghans secure their livelihood in Afghanistan, Iran and Pakistan. BICC-Working Paper, 2, BICC (Bonn International Center for Conversion), Bonn.

Griffiths M. (2014), Out of Time: The Temporal Uncertainties of Refused Asylum Seekers and Immigration Detainees. Journal of Ethnic and Migration Studies, 40 (12), pp. 1991-2009.

Griffiths M., Rogers A. \& Anderson B. (2013), Migration, Time and Temporalities: Review and Prospect. Oxford: Centre on Migration, Policy and Society (COMPAS) Research Resources Paper. 
Hainmueller J., Hangartner D. \& Lawrence D. (2016), When lives are put on hold: Lenghty asylum processes decrease employment among refugees. Science Advances, 2 (8). $<$ DOI: 10.1126/sciadv.1600432>, >https://advances.sciencemag.org/content/2/8/e1600432> [Zugriff: 11-04-2019].

Han C. (2018), Precarity, Precariousness, and Vulnerability. Annual Review of Anthropology, 47, pp. 331-343.

Harrell-Bond B.E. (1999), 'The Experience of Refugees as Recipients of Aid'. In: Ager A. (ed.): Refugees: Perspectives on the Experience of Forced Migration. London, Continuum International Publishing Group Ltd, pp. 136-168.

Heesen J., Lorenz D.F., Nagenborg M., Wenzel B. \& Voss M. (2014), Blind Spots on Achilles' Heel. The Limitations of Vulnerability and Resilience Mapping in Research. International Journal of Disaster Risk Science, 5 (1), pp. 74-85.

Hoffmaster B. (2006), What Does Vulnerability Mean? The Hastings Center Report, 36 (2), pp. 38-45.

Horst C. (2008), Transnational Nomads. How Somalis Cope with Refugee Life in the Dadaab Camps of Kenya. Oxford, Berghahn Books.

Houte M. van, Siegel M. \& Davids T. (2016), Deconstructing the meanings of and motivations for return: an Afghan case study. Comparative Migration Studies, 4 (21). <https://doi. org/10.1186/s40878-016-0042-y>.

Howden D. \& KodalaK M. (2018), The Vulnerability Contest. Blog auf: News Deeply/Refugees Deeply, 17-10-2018. <https://www.newsdeeply.com/refugees/articles/2018/10/17/thevulnerability-contest> [Zugriff: 28-01-2020].

HruschKa C. \& Leboeuf L. (2019), Vulnerability: a buzzword or a standard for migration governance? Population \& Policy Compact, Policy Brief, 20.

Hyndman J. (2000), Managing Displacement: Refugees and the Politics of Humanitarianism. Minneapolis, University of Minnesota Press.

Hyndman J. \& GiLes W. (2011), Waiting for what? The feminization of asylum in protracted situations. Gender, Place and Culture, A Journal of Feminist Geography, 18 (3), pp. 361379.

IOM (о.J.), A global compact for safe, orderly and regular migration. <https://www.iom.int/ global-compact-migration> [Zugriff: 12-07-2020].

IOM (2017), International Dialogue on Migration 2017. Understanding migrant vulnerabilities: A solution-based approach towards a global compact that reduces vulnerabilities and empowers migrants. Background paper. 18-19 July 2017. Palais des Nations, Geneva. $<$ https://www.iom.int/international-dialogue-migration-2017-understanding-migrantvulnerabilities-solution-based-approach> [Zugriff: 28-01-2020].

Jefrerey C. (2008), Guest editorial: Waiting. Environment and Planning D: Society and Space, 26, pp. 954-958.

Kapoor I. (2008), The Postcolonial Politics of Development. London, New York, Routledge.

Kasmir S. (2018), Precarity. In: Stein F., Lazar S., Candea M., Diemberger H., Robbins J., Sanchez A. \& Stasch R. (eds.): The Cambridge Encyclopedia of Anthropology, pp. 1-14. $\quad<$ http://doi.org/10.29164/18precarity>; $<$ https://www.researchgate.net/publica tion/323742243_Precarity/link/5b6f3299a6fdcc87df724c12/download $>$.

KhosRavi Sh. (2010), An Ethnography of Migrant 'Illegality` in Sweden: Included Yet Excepted? Journal of International Political Theory, 6 (1), pp. 95-66. 
Khosravi Sh. (2014), Waiting. In: Anderson B. \& Keith M. (eds.): Migration: The COMPAS Anthology. Oxford: ESRC Centre on Migration, Policy and Society (COMPAS), pp. 1-2. $<$ http:// compasanthology.co.uk/waiting/> [Zugriff: 20-02-2015].

Khosravi Sh. (ed.) (2018a), After Deportation. Ethnographic Perspectives. Cham: Palgrave Macmillan.

Khosravi Sh. (2018b), Introduction. In: KhosRavi Sh. (ed.), After Deportation. Ethnographic Perspectives. Cham, Palgrave Macmillan, pp. 1-14.

Khosravi Sh. (2018c), Stolen Time. Radical Philosophy, 2.03, 2, pp. 38-41. <https://www. radicalphilosophy.com/article/stolen-time>.

Khosravi Sh. (2019), EASA 2018 Keynote: What do we see if we look at the border from the other side? Social Anthropology/Anthropologie Sociale, 27 (3), pp. 409-424.

Kibreab G. (1993), The Myth of Dependency among Camp Refugees in Somalia 1979-1989. Journal of Refugee Studies, 4 (4), pp. 321-349.

KLeIST O.J. (2015), Über Flucht forschen. Herausforderungen der Flüchtlingsforschung, Peripherie, 138/139 (35), pp. 150-169.

KLEIST O.J. (2018), Mixed Migration: Der Flüchtling als politisch und ethische Figur, Ethik und Unterricht, 2, pp. 10-13.

KOHLBACHER J. (2019), “... Aber zu finden eine Wohnung ist so schwer!“ - Wohnintegration und „Vulnerablität“ Geflüchteter Afghan/inn/en sowie die kompensatorische Rolle sozialer Netzwerke. In: Kohlbacher J. \& Six-Hohenbalken M. (eds.), Die lange Dauer der Flucht - Analysen aus Wissenschaft und Praxis. Wien, Verlag der Österreichischen Akademie der Wissenschaften, pp. 143-169.

Kohli R.K.S. \& KaukKo M. (2018), The Management of Time and Waiting by Unaccompanied Asylum-Seeking Girls in Finland. Journal of Refugee Studies, 31 (4), pp. 488-506. <https:// doi.org/10.1093/jrs/fex040>.

Krause U. (2016), »It Seems You Don't Have Identity, You Don't Belong«. Reflexionen über das Flüchtlingslabel und dessen Implikationen. Zeitschrift für Internationale Beziehungen, 23 (1), pp. 8-37.

Krause U. \& Schmidt H. (2019), Refugees as Actors? Critical Reflections on Global Refugee Policies on Self-reliance and Resilience. Journal of Refugee Studies, fez059, pp. 22-41. $<\mathrm{https}: / /$ doi.org/10.1093/jrs/fez059>.

Labman Sh.E. (2012), At Law's Border: Unsettling Refugee Resettlement. PhD thesis, Faculty of Law, University of British Columbia, defended on 15 November 2012.

Lakraa H. (2017), Im/Mobility, Power, and In/Visible Refugees. Postcolonial Text, 12 (3 \& 4).

Lewis H., Dvyer P., Hodkinson S. \& Waite L. (2015), 'Hyper-precarious Lives: Migrants, Work and Forced Labour in the Global North'. Progress in Human Geography, 39 (5), pp. 580600.

Lilja M., Henriksson A. \& BaAz M. (2019), (Re)thinking the Precarity of Swedish Migrants: Governing through Decelerations and Timescapes. Journal of Refugee Studies, 32 (1), pp. 144-161. <https://doi.org/10.1093/jrs/fey021>.

LøNNING M.N. (2018), Fragmented journeys, social relations and age amongst Afghan young people on the move towards Europe. Positioning, negotiating and redefining. Ph.D. thesis, Trondheim January 2018. Norwegian University of Sciences and Technology, Faculty of Social and Educational Sciences, Department of Social Work. 
LoREnZ D.F. (2018), „All refugees are vulnerable“: Vulnerabilität, Konflikte und Katastrophen im Spiegel Postkolonialer Theorie. Zeitschrift für Friedens- und Konfliktforschung, Sonderband 2, pp. 60-98.

LoRey I. (2011), 'Governmental Precarization - Transversal' < http://eipcp.net/transversal/0811/ lorey/en/> [Zugriff: 23-03-2018].

LoRey I. (2015), State of Insecurity: Government of the Precarious. London/New York: Verso.

LudwiG B. (2013), "Wiping the Refugee Dust from My Feet”: Advantages and Burdens of Refugee Status and the Refugee Label, International Migration 54(1), pp. 5-18<DOI: 10.1111/imig.12111> [Zugriff: 20-08-2020].

Lyons K. (2017), 'Destitution is routine': refugees face homelessness even after asylum. The Guardian, 08-09-2017. <https://www.theguardian.com/world/2017/sep/08/destitution-isroutine-refugees-face-homelessness-even-after-gaining-asylum> [Zugriff: 15-07-2020].

MackenZie C., Rogers W. \& Dodds S. (eds.) (2014a), Vulnerability: New Essays in Ethics and Feminist Philosophy. Oxford, New York etc., Oxford University Press.

Mackenzie C., Rogers W. \& DodDs S. (2014b), Introduction. What Is Vulnerability, and Why Does It Matter for Moral Theory? In: Mackenzie C., Rogers W. \& Dodds S. (eds.), Vulnerability: New Essays in Ethics and Feminist Philosophy. Oxford, New York, etc., Oxford University Press, pp. 1-29 <DOI:10.1093/acprof:oso/9780199316649.003.0001>.

MajIDI N. (2018), Deportees Lost at "Home": Post-deportation Outcomes in Afghanistan. In: Khosravi Sh. (ed.), After Deportation. Ethnographic Perspectives. Cham, Palgrave Macmillan, pp. 127-148.

Malkki L.H. (1995a), Purity and Exile. Violence, Memory, and National Cosmology Among Hutu Refugees in Tanzania. Chicago, IL, Chicago University Press.

Malkki L.H. (1995b), Refugees and Exile. From >Refugee Studies to the National Order of Things. Annual Review of Anthropology, 24 (1), pp. 495-523.

MaLkKi L.H. (1996), Speechless Emissaries. Refugees, Humanitarianism, and Dehistoricization. Cultural Anthropology, 11 (3), pp. 377-404.

Marbach M., Hainmueller J. \& Hangartner D. (2018), The long-term impact of employment bans on the economic integration of refugees. Science Advances, 4 (9). <http://doi. org/10.1126/sciadv.aap9519>.

Mokre M. (2015), Solidarität als Übersetzung: Überlegungen zum Refugee Protest Camp Vienna. Wien, Linz, Berlin etc., transversal texts.

Monsutti A. (2008), Afghan Migratory Strategies and the Three Solutions to the Refugee Problem. Refugee Survey Quarterly, 27 (1), pp. 58-73.

Monsutti A. (2018), Mobility as a political act. Ethnic and Racial Studies, 41 (3), pp. 448-455.

Mountz A., Wright R., Miyares I. \& Bailey A.J. (2002), Lives in limbo: Temporary Protected Status and immigrant identities. Global Networks, 2 (4) , pp. 335-356.

Mulert C. (2019), Eine neue ,Reservearmee'? Ausbildungschancen von Geflüchteten in der Hotel- und Gastronomiebranche. IMIS Working Paper 04, Institut für Migrationsforschung und Interkulturelle Studien (IMIS) der Universität Osnabrück. IMIS: Osnabrück.

NAwyn St.J. (2012), Review: Michel Agier: Managing the Undesirables: Refugee Camps and Humanitarian Government (translated by David Fernbach), Cambridge, Polity. Contemporary Sociology, 41 (1), pp. 57-58.

Neve A. (2014), An Exploration of the Renegotiated and Performed Identities among Hazara Men in Australia. MA Thesis in Anthropology, The Australian National University. 
Newman E. (2003), Refugees, International Security, and Human Vulnerability. Introduction and Survey. In: Newman E. \& Selm J. van (eds.), Refugees and Forced Displacement. International Security, Human Vulnerability, and the State. Tokyo, New York, Paris, United Nations University Press, pp. 3-30.

Olivius E. (2016a), Constructing Humanitarian Selves and Refugee Others, International Feminist Journal of Politics, 18 (2), pp. 270-290.

Olivius E. (2016b), Refugee Men as Perpetrators, Allies or Troublemakers? Emerging Discourses on Men and Masculinities in Humanitarian Aid. Women's Studies International Forum, 56, pp. 56-65.

ONG A. (2003), Buddha Is Hiding: Refugees, Citizenship, the New America. Berkeley: University of California Press.

Parla A. (2019), Precarious Hope. Migration and the Limits of Belonging in Turkey. Stanford, Cal.: Stanford University Press.

Parpart J.L. (1995), Deconstructing the Development ıExpert<. Gender, Development and the >Vulnerable Groups . In: Marchand M.H. \& Parpart J.L. (eds.), Feminism/Postmodernism/ Development, London, Routledge, pp. 221-243.

PASCuCCI E. (2015), Chap. 10: Diaspora, Immobility and the Experience of Waiting: Young Iraqi Refugees in Cairo. In: Gorman A. \& Kasbarian S. (eds.), Diasporas of the Modern Middle East: Contextualising Community. Edinburgh: Edinburgh University Press, pp. 338-369.

Pervou I. (2017), Refugees and Vulnerability: The Crisis and the Shift in Human Rights Protection. QMHRR, 4 (11).

Picozza F. (2017), Dubliners: Unthinking Displacement, Illegality, and Refugeeness within Europe's Geographies of Asylum. In: De Genova N. (ed.), The Borders of "Europe". Autonomy of Migration, Tactics of Bordering. Durham and London, Duke University Press, pp. 233-254.

Polanco G. (2016), 'Consent behind the Counter: Aspiring Citizens and Labour Control under Precarious (Im)migration Schemes’. Third World Quarterly, 27 (8), pp. 1332-1350.

PunZ S. (2019), “Integration von Aslywerber_innen - ein Widerspruch? Eine qualitativeempirische Studie in drei ländlichen Nachb̄argemeinden im Mostviertel, Niederösterreich - unter besonderer Berücksichtigung des Aspekts Bildung”. MA-Thesis, KSA, Universität Wien.

RAJARAM P.K. (2002), Humanitarianism and Representations of the Refugees. Journal of Refugee Studies, 15 (3), pp. 247-264.

Reuter J. \& Villa P.-I. (2010), Provincializing Soziologie. Postkoloniale Theorie als Herausforderung. In: Reuter J. \& Villa P.-I. (eds.), Postkoloniale Soziologie. Empirische Befunde, theoretische Anschlüsse, politische Intervention. Bielefeld, Transcript Verlag, pp. $1-46$.

Rotter R. (2010), 'Hanging In-Between': Experiences of Waiting among Asylum Seekers Living in Glasgow. PhD Thesis in Social Anthropology, University of Edinburgh.

RotTER R. (2016), Waiting in the asylum determination process: Just an empty interlude? Time \& Society, 25 (1), pp. 80-101.

SCAlettaris G., Monsutti A. \& Donini A. (2019), Young Afghans at the doorsteps of Europe: the difficult art of being a successful migrant. Journal of Ethnic and Migration Studies. $<$ DOI: $10.1080 / 1369183$ X.2019.1618250>. 
SCHeibelhofer P. (2017), 'It won't work without ugly pictures': images of othered masculinities and the legitimisation of restrictive refugee-politics in Austria, NORMA, International Journal for Masculinity Studies, 12 (2), pp. 96-111. <https://doi.org/10.1080/18902138. 2017.1341222>.

SCHeibelhofer P. (2019), Gender and Intimate Solidarity in Refugee-Sponsorships of Unaccompanied Young Men. In: Feinschmidt M., Pries L. \& Cantat C. (eds.), Refugee Protection and Civil Society in Europe. Cham, Palgrave Macmillan, pp. 193-219.

Sigona N. (2014), The Politics of Refugee Voice. Representations, Narratives, and Memories. In: Fiddian-QAsmiyeh E., Loescher G., Long K. \& Sigona N. (eds.), The Oxford Handbook of Refugee and Forced Migration Studies. Oxford, Oxford University Press, pp. 369-382.

SPIvaK G.C. (2008), Can the Subaltern Speak? In: SpivaK G.C. (ed.): Can the Subaltern Speak? Postkolonialität und subalterne Artikulation, Wien [u.a.], Turia + Kant, pp. 17-118.

Standing G. (2014), The Precariat: The New Dangerous Class. New York, Bloomsbury Publishing.

Stewart E. (2005), Exploring the Vulnerability of Asylum Seekers in the UK. Population, Space and Place, 11, pp. 499-512.

Suliman S. \& Weber H. (2019), Global development and precarity: a critical political analysis. Globalizations, 16 (4), pp. 525-540.

TÄUBIG V. (2009), Totale Institution Asyl. Empirische Befunde zu alltäglichen Lebensführungen in der organisierten Desintegration. Weinheim, München, Juventa Verlag.

THE World BANK \& UNHCR (2011), Research Study on IDP's in Urban settings - Afghanistan. Kabul.

Tickтin M. (2017), A world without innocence. American Ethnologist, 44 (4), pp. 577-590. $<$ https://doi-org.uaccess.univie.ac.at/10.1111/amet.12558>.

Turner S. (2010), Politics of Innocence. Hutu Identity, Conflict and Camp Life. New York, NY, Berghahn Books.

UN-Generalversammlung (2016), Resolution der Generalversammlung, verabschiedet am 19. September 2016. 71/1 New Yorker Erklärung für Flüchtlinge und Migranten. 3. Oktober 2016, Einundsiebzigste Tagung, Tagesordnungspunkte 13 und 117. <https://www.un.org/ depts/german/gv-71/band1/ar71001.pdf> [Zugriff: 12-07-2020].

UN-GeneralVersammlung (2018), Bericht des Hohen Flüchtlingskommissars der Vereinten Nationen Teil II Globaler Pakt für Flüchtlinge. Generalversammlung Offizielles Protokoll Dreiundsiebzigste Tagung Beilage 12, A/73/12 (Part II), United Nations, New York. $<$ https://www.unhcr.org/dach/wp-content/uploads/sites/27/2018/11/GCR_final_GER.pdf > [Zugriff: 12-07-2020].

UNHCR (o.J.), The Global Compact on Refugees. <https://www.unhcr.org/the-global-compacton-refugees.html> [Zugriff: 12-07-2020].

UNHCR (2016a), New York Declaration for Refugees and Migrants. [https://www.unhcr.org/ new-york-declaration-for-refugees-and-migrants.html; Zugriff 12-07-2020].

UNHCR (2016b), New York Declaration for Refugees and Migrants. UNHCR's Quick Guide on New York Declaration for Refugees and Migrants. <https://www.unhcr.org/57e4f6504> [Zugriff: 12-07-2020].

UNHCR, InTERNATIONAL DETENTION COALITION(IDC) \& OAK FoundaTION (2016), Vulnerability Screening Tool. Identifying and addressing vulnerability: a tool for Asylum and Migration Systems.Geneva. <https://www.unhcr.org/protection/detention/57fe30b14/unhcr-idc-vulnera bility-screening-tool-identifying-addressing-vulnerability.html> [Zugriff: 12-07-2020]. 
United Nations Human Rights Council (2017), Resolution on the Protection of the Human Rights of Migrants: The Global Compact for Safe, Orderly and Regular Migration, 22 June 2017, A/HRC/RES/35/17, para. 18(b). <https://www.un.org/en/development/desa/ population/migration/generalassembly/docs/globalcompact/A_HRC_RES_35_17.pdf $>$ [Zugriff: 12-07-2020].

UN National Assembly (2018), Seventy-third session, Agenda items 14 and 119. Resolution adopted by the General Assembly on 19 December 2018 [without reference to a Main Committee (A/73/L.66)], 73/195. Global Compact for Safe, Orderly and Regular Migration, Distr. General 11 January 2019. <https://www.un.org/en/ga/search/view_doc. asp?symbol=A/RES/73/195> [Zugriff: 12-07-2020].

Villegas F.J. (2010), Strategic In/Visibility and Undocumented Migrants. In: DeI G. \& Simmons M. (eds.): Fanon and education: Thinking through Pedagogical Possibilities. New York, Peter Lang Publishing Group, pp. 147-170.

Wyss A. (2018), Illegalisation, Masculinity and Intimacy. The Impact of Public Images on Male Migrants with a Precarious Legal Status. Tsantsa, Revue de la Society Suisse d'Ethnologie, 23, pp. 121-126.

Wyss A. (2019), Stuck in Mobility? Interrupted Journeys of Migrants With Precarious Legal Status in Europe. Journal of Immigrant \& Refugee Studies, 17 (1), pp. 77-93. <https://doi. org/10.1080/15562948.2018.1514091>.

Yaghmainn B. (2016), We Must Redefine Vulnerability in the 2018 Refugee Compact. Blog auf: News Deeply/Refugees Deeply, published 14-10-2016. <https://www.newsdeeply.com/ refugees/community/2016/10/14/on-the-road-to-2018-we-must-redefine-vulnerabilitypart-1> [Zugriff: 20-01-2020].

Zetter R. (1991), Labelling Refugees. Forming and Transforming a Bureaucratic Identity. Journal of Refugee Studies, 4 (1), pp. 39-62.

Zetter R. (2007), More Labels, Fewer Refugees: Remaking the Refugee Label in an Era of Globalization, Journal of Refugee Studies, 20 (2), pp. 172-192. 